\title{
EZH2 oncogenic mutations drive epigenetic, transcriptional, and structural changes within chromatin domains
}

\author{
Maria C. Donaldson-Collier ${ }^{1,5}$, Stephanie Sungalee ${ }^{1,5}$, Marie Zufferey ${ }^{2,3,5}$, Daniele Tavernari ${ }^{2,3,5}$,

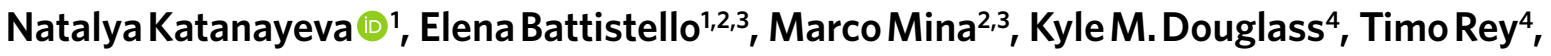 \\ Franck Raynaud ${ }^{2,3}$, Suliana Manley ${ }^{(4}{ }^{4}$, Giovanni Ciriello $\mathbb{1}^{2,3 \star}$ and Elisa Oricchio ${ }^{1 \star}$
}

\begin{abstract}
Chromatin is organized into topologically associating domains (TADs) enriched in distinct histone marks. In cancer, gain-offunction mutations in the gene encoding the enhancer of zeste homolog 2 protein (EZH2) lead to a genome-wide increase in histone-3 Lys27 trimethylation (H3K27me3) associated with transcriptional repression. However, the effects of these epigenetic changes on the structure and function of chromatin domains have not been explored. Here, we found a functional interplay between TADs and epigenetic and transcriptional changes mediated by mutated EZH2. Altered EZH2 (p.Tyr646* (EZH2 ${ }^{\mathrm{Y} 646 \mathrm{X})}$ ) led to silencing of entire domains, synergistically inactivating multiple tumor suppressors. Intra-TAD gene silencing was coupled with changes of interactions between gene promoter regions. Notably, gene expression and chromatin interactions were restored by pharmacological inhibition of EZH2 ${ }^{\mathrm{Y} 646 \mathrm{X}}$. Our results indicate that EZH2 ${ }^{\mathrm{Y} 646 \mathrm{X}}$ alters the topology and function of chromatin domains to promote synergistic oncogenic programs.
\end{abstract}

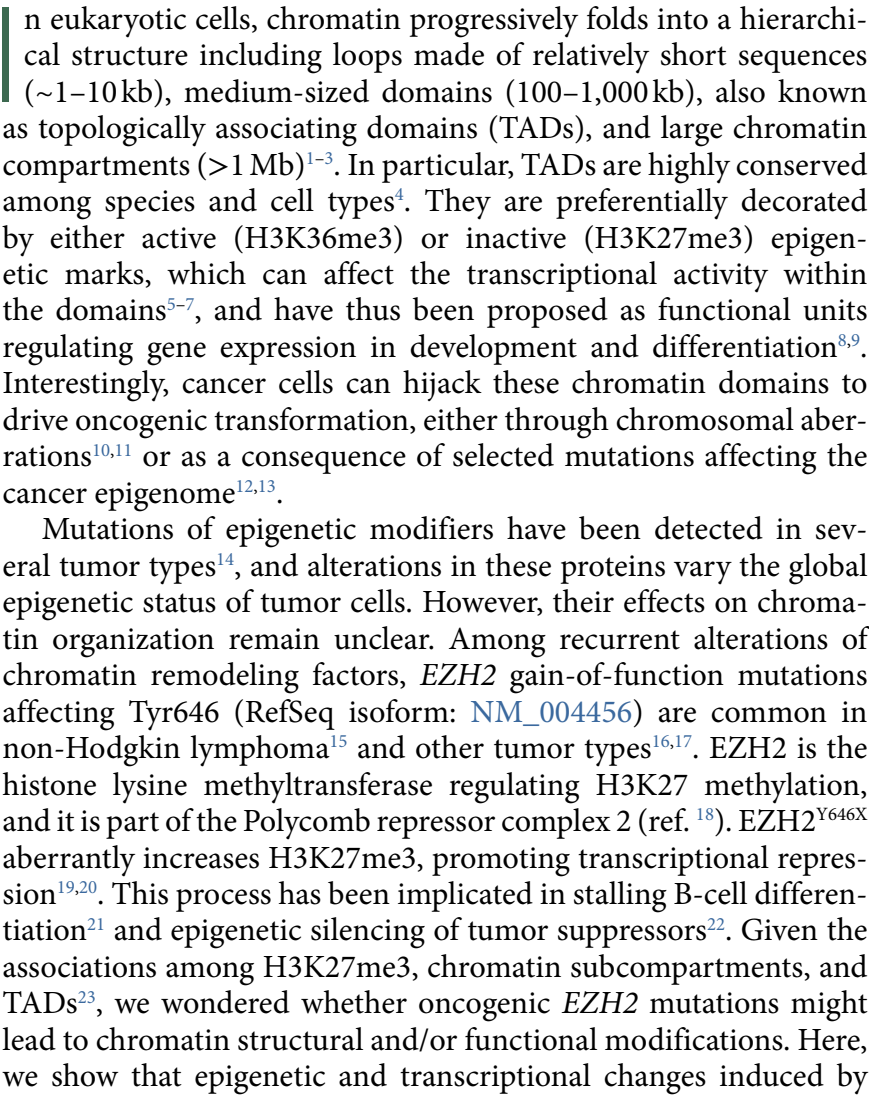

EZH2 mutations are more concordant within TADs than expected and modulate intra-TAD interactions proximal to gene promoters. Intra-TAD structural changes are associated with transcriptional inactivation of entire domains. Our results illustrate how cancerassociated epigenetic alterations can act beyond single genes and modify the activity of entire chromatin domains.

\section{Results}

$\mathrm{Hi}-\mathrm{C}$ chromatin maps of EZH2 ${ }^{\mathrm{WT}}$ and $\mathrm{EZH}^{\mathrm{Y} 646 \mathrm{X}}$ lymphomas. EZH2 p.Tyr646* gain-of-function alterations lead to a genomewide increase in H3K27me3 (Supplementary Fig. 1a). To establish whether this global accumulation of H3K27me3 modifies the genome topology on a similarly broad scale, we performed high-throughput chromatin conformation capture (Hi-C) in two lymphoma cell lines (Karpas422 and WSU-DLCL2) expressing the mutant form of $\mathrm{EZH} 2\left(\mathrm{EZH} 2^{\mathrm{Y} 646 \mathrm{X}}\right)$ and a lymphoma cell line (OCI-Ly19) expressing the wild-type EZH2 (EZH2 ${ }^{\mathrm{WT}}$ ) protein (Supplementary Table 1 and Supplementary Note). Contact maps of $\mathrm{EZH} 2^{\mathrm{Y} 646 \mathrm{X}}$ and $\mathrm{EZH} 2^{\mathrm{WT}}$ cells were binned in regions of $50 \mathrm{~kb}$ and compared with multiple metrics (Fig. 1). For each pair of maps, we compared the overall distribution of chromosomal contacts by using the stratum-adjusted correlation coefficient (SCC $)^{24}$ (Fig. 1a); the fraction of $1-\mathrm{Mb}$ regions assigned to the same compartment $(\mathrm{A} \text { or } \mathrm{B})^{3}$ (Fig. 1b); the similarity among TADs ${ }^{25-27}$ (Fig. 1c); and the fraction of bin interactions that were significant in both maps, also known as the cell interactome ${ }^{28,29}$ (Fig. 1d). To build a reference scale of values for each metric, we compared $\mathrm{Hi}-\mathrm{C}$ maps of $\mathrm{EZH} 2^{\mathrm{Y} 646 \mathrm{X}}$ lymphoma cell lines with $\mathrm{Hi}-\mathrm{C}$ maps of endothelial cells (HUVEC), fetal fibroblasts (IMR90), and normal lymphoblastoid cells (GM12878). Moreover, we used randomized contact maps or

'Swiss Institute for Experimental Cancer Research (ISREC), School of Life Science, École Polytechnique Fédérale de Lausanne (EPFL), Lausanne, Switzerland. ${ }^{2}$ Department of Computational Biology, University of Lausanne (UNIL), Lausanne, Switzerland. ${ }^{3}$ Swiss Institute of Bioinformatics (SIB), Lausanne, Switzerland. ${ }^{4}$ Institute of Physics, École Polytechnique Fédérale de Lausanne (EPFL), Lausanne, Switzerland. ${ }^{5}$ These authors contributed equally: Maria C. Donaldson-Collier, Stephanie Sungalee, Marie Zufferey, Daniele Tavernari. *e-mail: giovanni.ciriello@unil.ch; elisa.oricchio@epfl.ch 


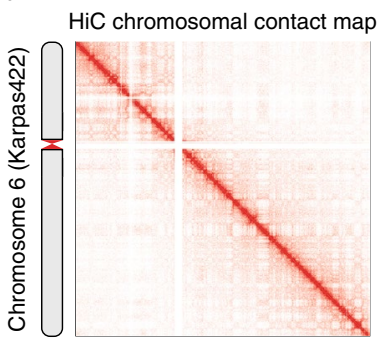

HiC contacts b

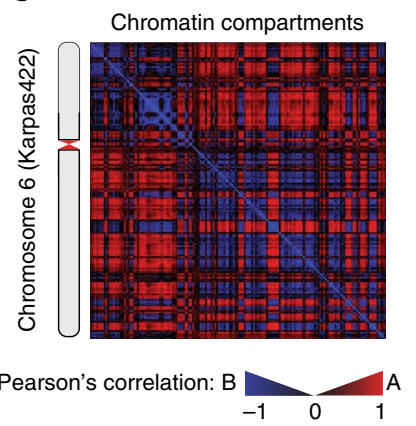

f

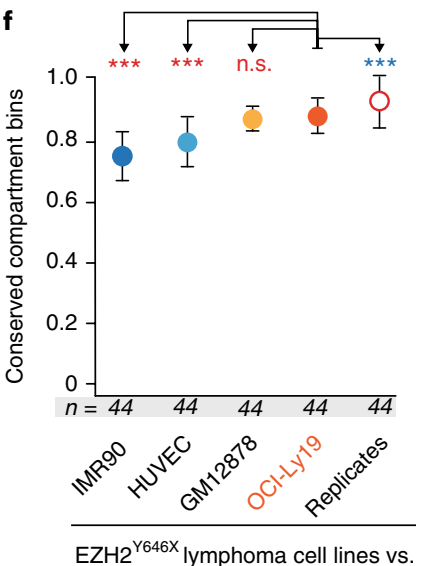

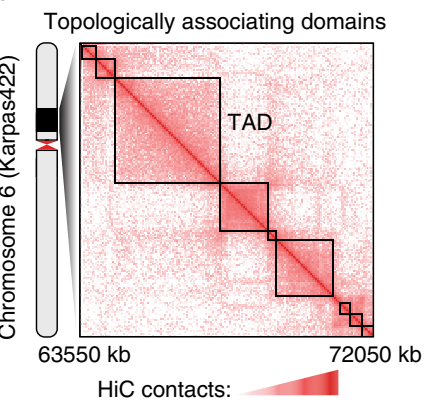

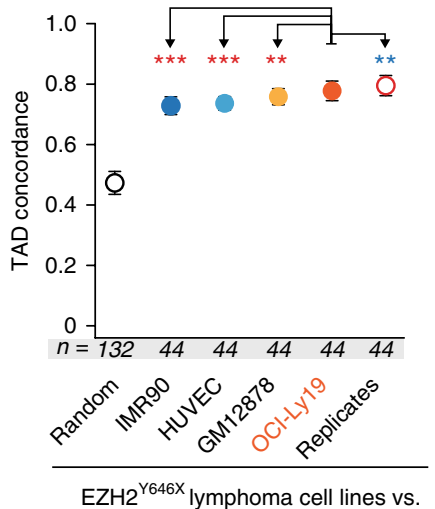

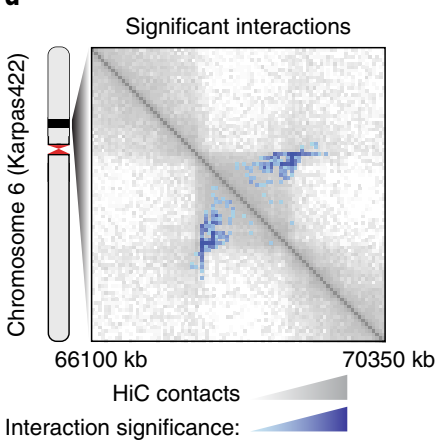

h i

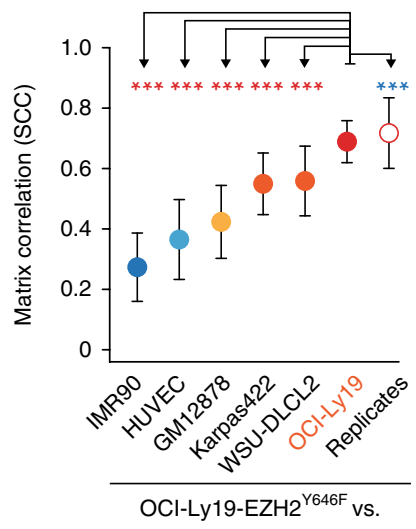

j

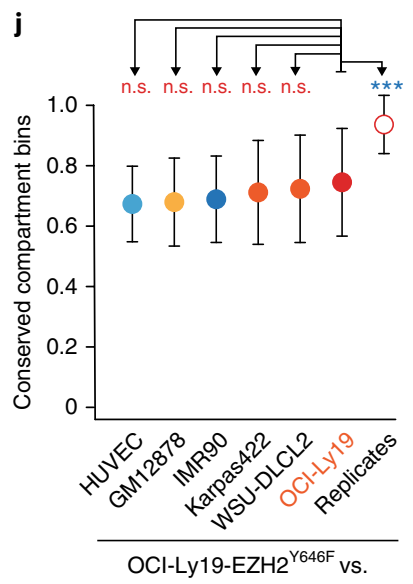

k

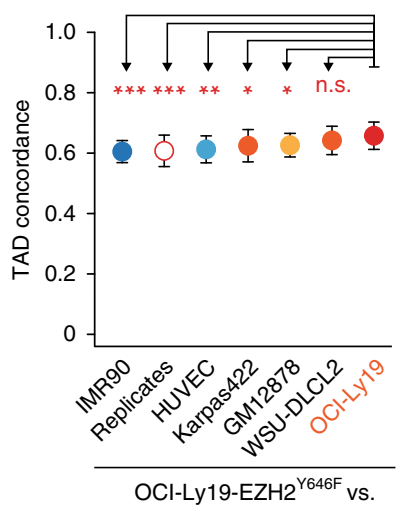

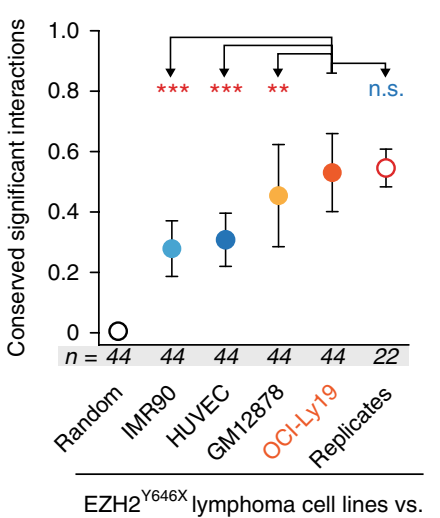

I

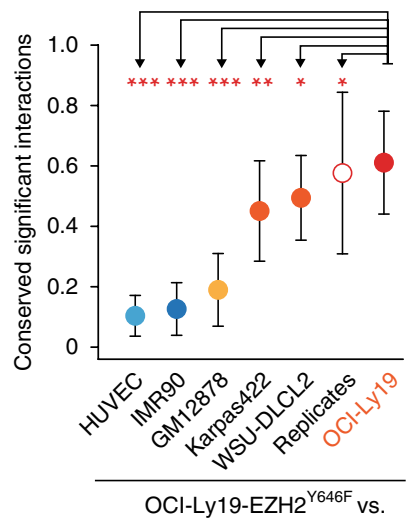

Fig. 1 | Comparison of the genome 3D organization in EZH2 ${ }^{\mathrm{WT}}$ and $\mathrm{EZH} 2^{\mathrm{Y} 646 \mathrm{X}}$ cells. a-d, Chromatin structural elements identifiable by Hi-C; representative images for Chr.6 of the GM12878 cell line: intrachromosomal contact map (a); correlation of observed/expected contact ratios for each pair of 1-Mb bins, with the plaid (blue/red) pattern showing chromatin compartments A or B (b); TADs (c); significant interactions determined by using HiC-DC, with all pairs of 50-kb bins within a 2-Mb window tested (d). e-h, Comparison between the intrachromosomal contact maps of EZH2 ${ }^{\mathrm{Y} 646 \mathrm{X}}$ cell lines (Karpas422 and WSU-DLCL2) and of the indicated cell lines, based on the overall Hi-C matrix correlation using the SCC (e); fraction of 1-Mb bins assigned to the same compartment (f); TAD similarity measured by measure of concordance $(\mathrm{MoC})(\mathbf{g})$; and fraction of shared significant interactions between $50-\mathrm{kb}$ bins determined by HiC-DC (h). $\mathbf{i}-\mathbf{I}$, Comparison between intrachromosomal contact maps of OCl-Ly19-EZH2 ${ }^{\mathrm{Y} 646 \mathrm{~F}}$ and of the indicated cell lines as described above, that is, based on SCC (i), compartment conservation (j), TAD similarity $(\mathbf{k})$, and the fraction of shared significant interactions (I). For all comparisons, the number of compared intrachromosomal maps is indicated below the graphs $(n)$, when not indicated $n=22$; the dotd represent the mean values, and error bars are \pm 1 standard deviation. $P$ values were computed with two-tailed Wilcoxon test (Supplementary Table 1 ). ${ }^{\star} P<0.05,{ }^{\star \star} P<0.01$, ${ }^{\star \star \star} P<0.001 ;$ n.s., not significant.

TADs and independent experimental replicates of EZH2 ${ }^{\mathrm{Y} 646 \mathrm{X}}$ lymphoma cell lines to provide the lowest and highest expected values for each metric, respectively. The similarity ranks obtained with all metrics were highly concordant and invariably showed that the contact maps from $\mathrm{EZH} 2^{\mathrm{Y} 646 \mathrm{X}}$ lymphoma were on average more similar to those from EZH $2^{\mathrm{WT}}$ cell lines than to any other cell model that we tested, except for their replicates (Fig. 1e-h and Supplementary Fig. 1b-e).

To validate this observation and allay nonspecific effects due to heterogeneity among samples, we generated a syngeneic lymphoma cell line (OCI-Ly19) expressing the altered protein $\mathrm{EZH} 2^{\mathrm{Y} 646 \mathrm{~F}}$ (c.1937A $>$ T, p.Tyr646Phe). We confirmed that expression of 
$\mathrm{EZH} 2^{\mathrm{Y} 646 \mathrm{~F}}$ increased $\mathrm{H} 3 \mathrm{~K} 27 \mathrm{me} 3$ across the genome, as compared with both the parental cell line and OCI-Ly19 transduced with an empty vector, whereas expression of EZH2 remained similar in the three conditions (Supplementary Fig. 1f,g). Hi-C contact maps derived for OCI-Ly19-EZH2 ${ }^{\mathrm{Y} 646 \mathrm{~F}}$ and OCI-Ly19 cells were as similar to or more similar than maps derived from independent replicates of the same cell line (Fig. 1i-l and Supplementary Fig. 1h). We conclude that although a certain diversity exists for a limited number of specific contacts and contact domains, on average, the genome topology of EZH2-mutated and wild-type lymphoma cells is highly similar.

EZH2 ${ }^{\mathrm{Y} 646 \mathrm{X}}$ inactivates selected TADs. Given the observed consistency between the chromatin structures of EZH2 ${ }^{\mathrm{Y} 646 \mathrm{X}}$ and $\mathrm{EZH} 2^{\mathrm{WT}}$ cell lines, we asked whether an association might exist between chromatin structural elements and the epigenetic and transcriptional changes induced by EZH2 gain-of-function mutations. In particular, we focused on chromatin subcompartments and TADs, given that both have been associated with H3K27me3 (ref. ${ }^{23}$ ).

To assess changes in $\mathrm{H} 3 \mathrm{~K} 27 \mathrm{me} 3$ specifically induced by EZH2 mutations, we analyzed H3K27me3 levels in OCI-Ly19 cells expressing either $\mathrm{EZH} 2^{\mathrm{Y} 646 \mathrm{~F}}$ or EZH2 ${ }^{\mathrm{WT}}$. The distribution of H3K27me3 in chromatin subcompartments confirmed the previously reported enrichment in $\mathrm{H} 3 \mathrm{~K} 27 \mathrm{me} 3$ within the B1 compartment $^{23}$ (Supplementary Fig. 2a). However, in OCI-Ly19-EZH2 ${ }^{\mathrm{Y} 646 \mathrm{~F}}$, we observed a significant but similar increase in H3K27me3 across all subcompartments (Supplementary Fig. 2a,b), thus suggesting that $\mathrm{EZH} 2^{\mathrm{Y} 646 \mathrm{X}}$ does not induce epigenetic changes specifically within one subcompartment.

Next, we tested whether differences in $\mathrm{H} 3 \mathrm{~K} 27 \mathrm{me} 3$ and gene expression were more concordant within TADs than expected and whether this association might lead to the activation or inactivation of specific domains (Fig. 2a). First, we extracted a consensus list of 2,038 TADs that comprised at least three expressed genes and were conserved among three EZH2 ${ }^{\mathrm{Y} 646 \mathrm{X}}$ cell lines (Karpas422, WSU-DLCL2, and OCI-Ly19-EZH2 $\left.{ }^{\mathrm{Y} 646 \mathrm{~F}}\right)$ and one EZH2 ${ }^{\mathrm{WT}}$ cell line (OCI-Ly19) (Supplementary Table 2). H3K27me3 chromatin immunoprecipitation and high-throughput sequencing (ChIP-seq) data were compared both between OCI-Ly19-EZH2 ${ }^{\text {WT }}$ and OCILy19-EZH2 ${ }^{\mathrm{Y} 646 \mathrm{~F}}$ and between OCI-Ly19-EZH2 ${ }^{\mathrm{WT}}$ and two cell lines (Karpas422 and WSU-DLCL2) with endogenous EZH2 mutations. The TAD mean H3K27me3 values in OCI-Ly19-EZH2 ${ }^{\mathrm{Y} 646 \mathrm{~F}}$ were highly correlated with those observed in Karpas422 and WSUDLCL2 (Supplementary Fig. 2c); thus, we decided to focus on the OCI-Ly19-EZH2 ${ }^{\text {WT }}$ and OCI-Ly19-EZH2 ${ }^{\text {Y646F }}$ ChIP-seq datasets, because they reflected $\mathrm{H} 3 \mathrm{~K} 27 \mathrm{me} 3$ changes directly associated with the $E Z H 2$ mutation. The distribution of TAD mean $\mathrm{H} 3 \mathrm{~K} 27 \mathrm{me} 3 \mathrm{val}-$ ues exhibited a positive shift in $\mathrm{EZH} 2^{\mathrm{Y} 646 \mathrm{X}}$ compared with $\mathrm{EZH} 2^{\mathrm{WT}}$ cells (Fig. 2b). H3K27me3 profiles of loci within the same TAD were more correlated than profiles of loci belonging to distinct adjacent domains in both cell lines (Supplementary Fig. 2d,e). Moreover, H3K27me3 fold changes between OCI-Ly19 mutated cells and wild-type cells were also more correlated within the same TAD than between adjacent TADs (Fig. 2c), and this trend was independent of the distance between the genomic loci (Fig. 2d). Overall, these results indicate that the EZH2-mediated increase in $\mathrm{H} 3 \mathrm{~K} 27 \mathrm{me} 3$ is associated with the compartmentalization of the chromatin in TADs.

Interestingly, the distribution of $\mathrm{H} 3 \mathrm{~K} 27 \mathrm{me} 3$ fold changes $\left(\mathrm{EZH} 2^{\mathrm{Y} 646 \mathrm{X}}\right.$ versus $\left.\mathrm{EZH} 2^{\mathrm{WT}}\right)$ within TADs exhibited a greater number of high fold changes than random distributions obtained by permuting $50-\mathrm{kb}$ or $100-\mathrm{kb}$ intervals of $\mathrm{H} 3 \mathrm{~K} 27 \mathrm{me} 3 \mathrm{ChIP}$-seq reads (Fig. 2e). This trend was independent of the subcompartment to which each TAD was assigned (Supplementary Fig. 2f) and of the interval size used in the permutations (Supplementary Fig. 2g). Moreover, by comparing matched TAD mean H3K27me3 values in $\mathrm{EZH} 2^{\mathrm{WT}}$ and EZH2 ${ }^{\mathrm{Y} 646 \mathrm{~F}}$ OCI-Ly19 cells, we found that H3K27me3 did not proportionally increase in all domains, but fold changes were greater in domains that had low histone methylation in EZH2 ${ }^{\mathrm{WT}}$ cells than in domains that were already enriched in H3K27me3 (Fig. 2f). Therefore, the increase in H3K27me3 mediated by EZH2 alterations within specific domains was not randomly distributed but were dependent on the initial level of H3K27me3 (Fig. 2g).

Next, we assessed the effects of $\mathrm{H} 3 \mathrm{~K} 27 \mathrm{me} 3$ changes on transcription, in OCI-Ly19-EZH2 ${ }^{\mathrm{Y} 646 \mathrm{~F}}$ and OCI-Ly19-EZH2 ${ }^{\mathrm{WT}}$ cell lines. In particular, we wondered whether the observed correlation between H3K27me3 changes within TADs might be reflected in concordant mRNA expression changes in genes within the same TAD. For this purpose, we scored each TAD on the basis of the number and magnitude of concordant gene fold changes within the domain (mRNA fold-change concordance (FCC) scores; Supplementary Fig. 3a). For this analysis, we selected only TADs for which at least three genes had detectable mRNA expression in either OCI-Ly19$\mathrm{EZH} 2^{\mathrm{Y} 646 \mathrm{~F}}$ or OCI-Ly19-EZH2 ${ }^{\mathrm{WT}}$. The observed FCC scores were compared with those obtained after randomly permuting geneto-TAD assignments. The observed FCC scores were higher than expected on the basis of random permutations, thus indicating that changes in gene expression in OCI-Ly19-EZH2 ${ }^{\mathrm{Y} 646 \mathrm{~F}}$ compared with OCI-Ly19-EZH2 ${ }^{\text {WT }}$ were significantly concordant within TADs (Supplementary Fig. 3b).

To validate this finding in larger cohorts of B-cell lymphoma samples, we collected independent mRNA expression data for diffuse large B-cell lymphoma (DLBCL) cell lines ${ }^{30}$ including Karpas422 and WSU-DLCL2 (EZH2 ${ }^{\mathrm{WT}} n=2, \mathrm{EZH} 2^{\mathrm{Y} 646 \mathrm{X}} n=6$; each sample was analyzed in duplicate, GSE40792), and primary samples from germinal center (GC) patients with $\operatorname{DLBCL}^{21}\left(\mathrm{EZH}^{\mathrm{WT}} n=30\right.$,

Fig. 2 | Epigenetic and transcriptional changes in EZH2 ${ }^{\mathrm{Y} 646 \mathrm{X}}$ cells occur within TADs. a, Study design: H3K27me3 ChIP-seq, mRNA expression, and Hi-C data in $\mathrm{EZH} 2^{\mathrm{Y} 646 \mathrm{X}}$ and $\mathrm{EZH} 2^{\mathrm{WT}}$ lymphomas were integrated to identify epigenetically and transcriptionally inactive, neutral, and active TADs. $\mathbf{b}$, Distribution of mean H3K27me3 signal within TADs in OCl-Ly19-EZH2 ${ }^{\mathrm{WT}}$ and OCl-Ly19-EZH2 ${ }^{\mathrm{Y} 646 \mathrm{~F}}$ cells. c, Correlation across $n=2,038 \mathrm{TADs}$ of H3K27me3 fold changes in loci within the same TAD and loci separated by one TAD boundary. $\mathbf{d}$, Correlation ( $y$ axis) of H3K27me3 fold changes between all 25-kb bin pairs within the same TADs and bin pairs separated by one TAD boundary as a function of their distance ( $x$ axis). e, Distributions of mean H3K27me3 fold changes for $n=2,038$ TADs and of fold changes obtained by permuting H3K27me3 bins ( $50 \mathrm{~kb}$ and $100 \mathrm{~kb}$ ). f, Correlation between TAD mean H3K27me3 ( $n=2,038$ TADs) in OCl-Ly19-EZH2 ${ }^{\text {WT }}$ ( $x$ axis) and OCl-Ly19-EZH2 ${ }^{\text {Y646F }}$ ( $y$ axis). g, TAD mean H3K27me3 fold changes ( $n=2,038$ TADs) between OCl-Ly19-EZH2 ${ }^{\mathrm{Y} 646 \mathrm{~F}}$ and OCl-Ly19-EZH2 ${ }^{\mathrm{WT}}$; H3K27me3 values in OCI-Ly19-EZH2 ${ }^{\mathrm{WT}}$ were binned $\left(\right.$ bin $_{i}=(i-1, i)$ ), and TAD mean H3K27me3 fold changes in each bin are reported. Expected values were determined by TAD permutation. $\mathbf{h}-\mathbf{j}$, Cumulative sum curves of mRNA FCC for EZH2 ${ }^{\mathrm{Y} 646 \mathrm{X}}(n=12)$ versus EZH2 ${ }^{\text {WT }}$ cells $(n=4)$ in 2,038 TADs $(\mathbf{h}), \mathrm{GC}-\mathrm{DLBCL} \mathrm{EZH} 2^{\mathrm{Y} 646 \mathrm{X}}(n=7)$ versus EZH2 ${ }^{\mathrm{WT}}(n=30)$ patient samples in 2,038 TADs (i), and FL EZH2 ${ }^{\mathrm{Y} 646 \mathrm{X}}$ $(n=6)$ versus EZH2 ${ }^{\mathrm{WT}}(n=17)$ patient samples in 1,908 TADs $(\mathbf{j})$. Observed curves are compared with random curves obtained after 10,000 gene-to-TAD assignment permutations (gray area delimits minimum and maximum values; dark line is the mean). $\mathbf{k}, \mathbf{l}$, TAD mean H3K27me 3 fold changes between OCl-Ly19-EZH2 ${ }^{\mathrm{Y} 646 \mathrm{~F}}$ and OCl-Ly19-EZH2 ${ }^{\mathrm{WT} T}$ cells ( $x$ axis) versus the TAD mRNA-expression fold changes in EZH2 ${ }^{\mathrm{Y} 646 \mathrm{X}}$ versus EZH $2^{\mathrm{WT}}$ cell lines ( $y$ axis) for $n=2,038$ TADs; in $\mathbf{I}$, inactive TADs $\left(\log _{2}\left(\mathrm{FC}_{\mathrm{H} 3 \mathrm{k} 27 \mathrm{me} 3}\right)>1\right.$ and $\left.\log _{2}\left(\mathrm{FC}_{\mathrm{mRNA}}\right)<-1\right)$ are highlighted. $\mathbf{m}$, Gene-set enrichment analysis for $n=283$ genes within inactive TADs according to Gene Ontology categories $(n=5,337)$ and experimentally derived gene sets $(n=3,409)$. Gene-set enrichment was tested by hypergeometric test (one-sided) and adjusted by false discovery rate. 
$\left.\mathrm{EZH} 2^{\mathrm{Y} 646 \mathrm{X}} n=7, \mathrm{GSE} 23501\right)$ and patients with follicular lymphoma $(\mathrm{FL})^{31}\left(\mathrm{EZH} 2^{\mathrm{WT}} n=17, \mathrm{EZH} 2^{\mathrm{Y} 646 \mathrm{X}} n=6\right.$, PRJNA278311). Across all datasets, we verified that mRNA expression was more correlated between genes in the same TAD than between genes in different TADs, independently of their genomic distance (Supplementary Fig. 3c-e). Furthermore, the FCC scores obtained by comparing $\mathrm{EZH} 2^{\mathrm{Y} 646 \mathrm{X}}$ and $\mathrm{EZH} 2^{\mathrm{WT}}$ cases were always greater than random, with an overall increase ranging between 15\% and 33\% (increase in area under the curve; Fig. $2 \mathrm{~h}-\mathrm{j}$ and Supplementary Fig. $3 \mathrm{~b}$ ). Across all the expression datasets that we analyzed, expression changes associated with EZH2 mutations were more concordant within TADs than expected.

Next, we compared matched mean H3K27me3 and mRNA expression changes within TADs in OCI-Ly19-EZH2 ${ }^{\mathrm{Y} 646 \mathrm{~F}}$ and a

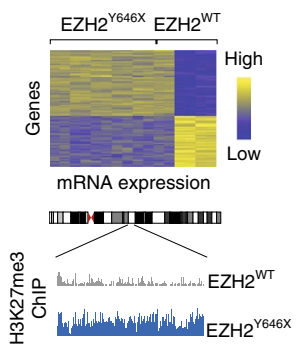

b

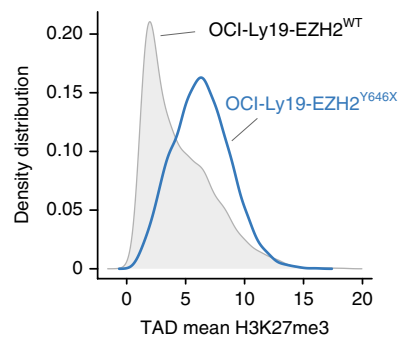

e

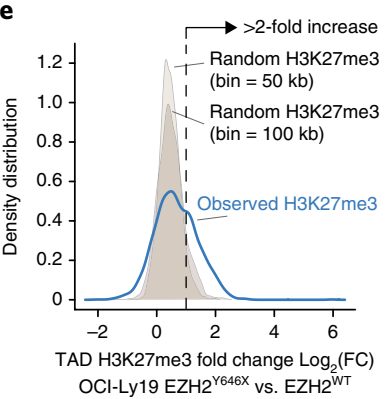

h

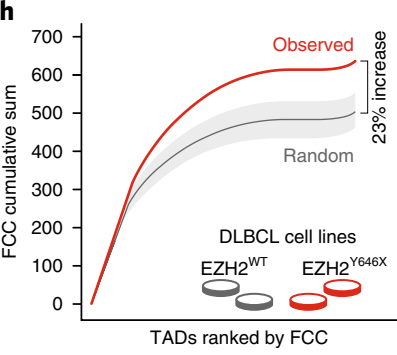

k

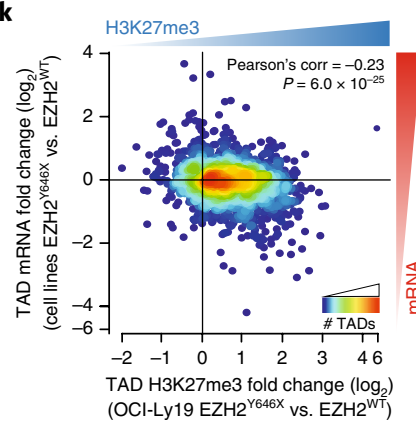

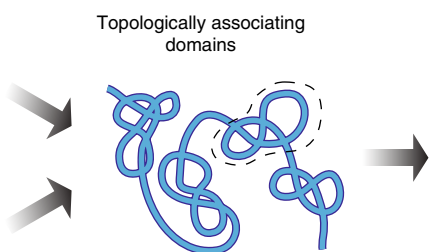

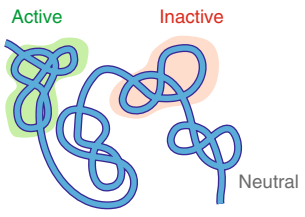

c

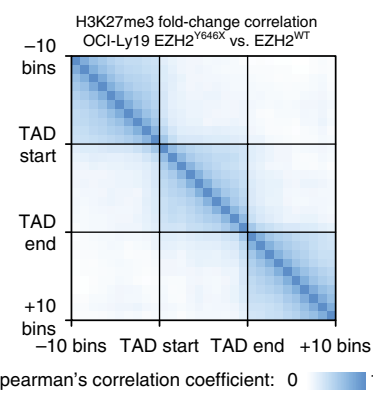

d

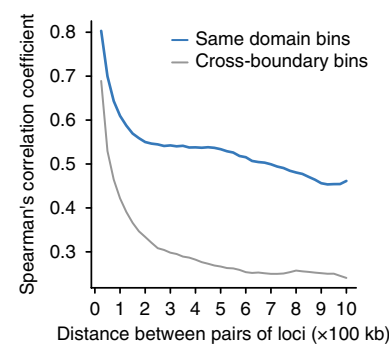

f

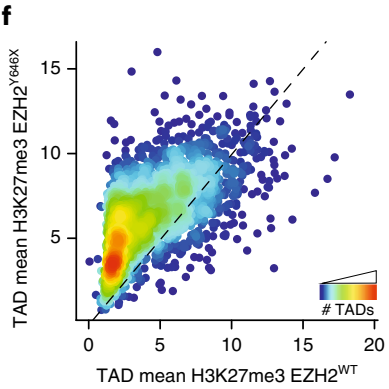

g
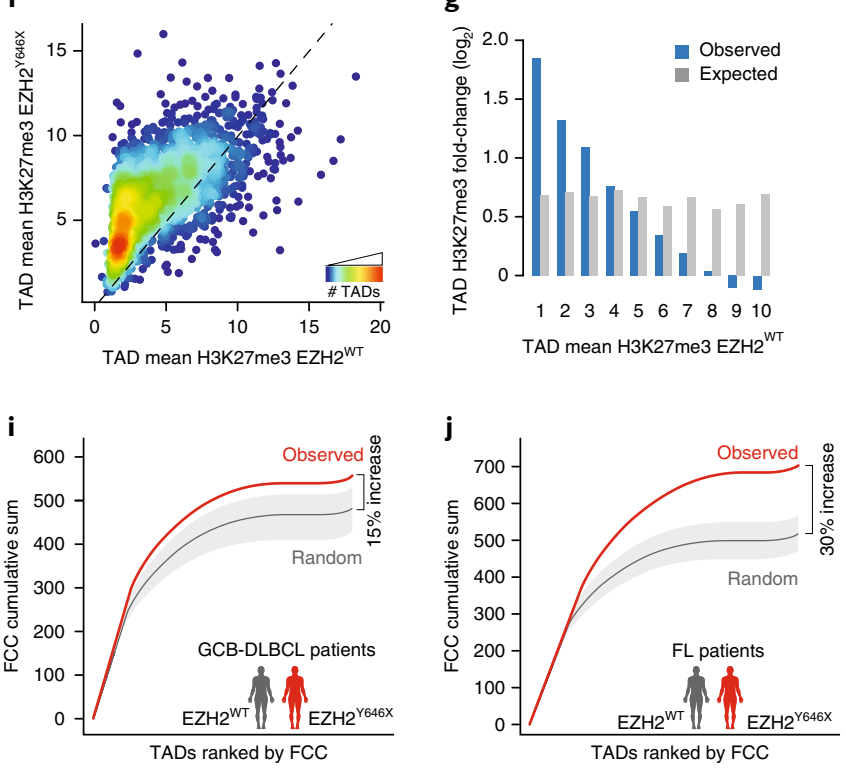

I

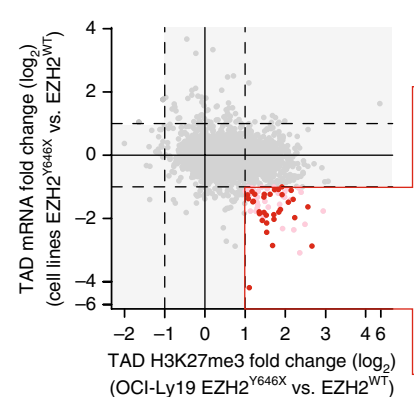

Inactive TADs [ $\begin{aligned} & \text { Stringent }(n=32) \\ & \text { Permissive }(n=40)\end{aligned}$ Other TADs $\quad(n=1,966)$

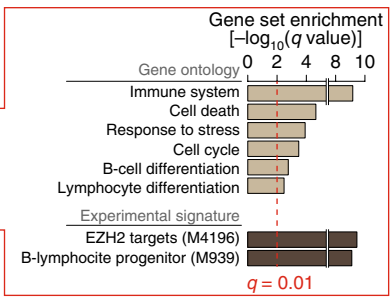




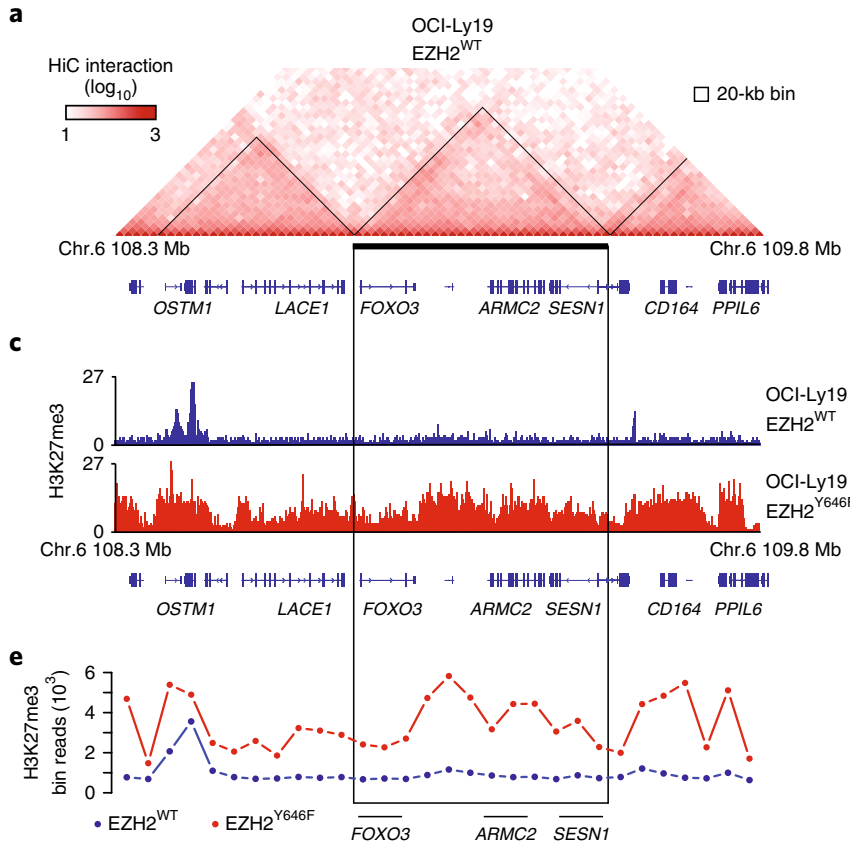

$\mathbf{g}$
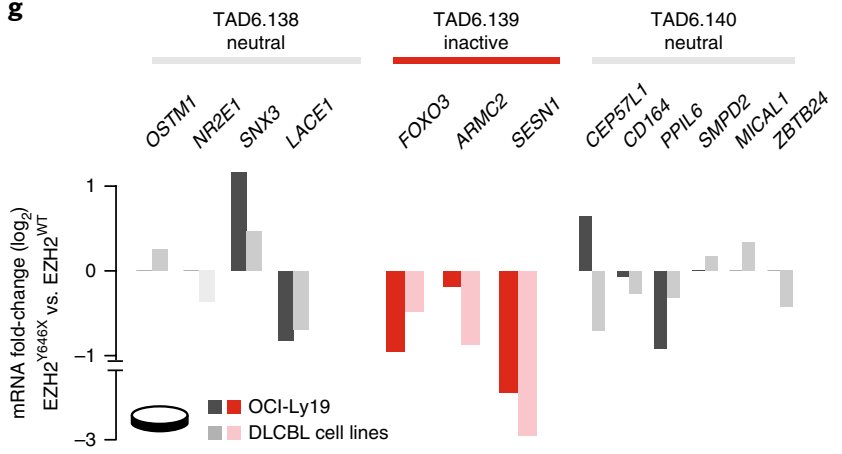

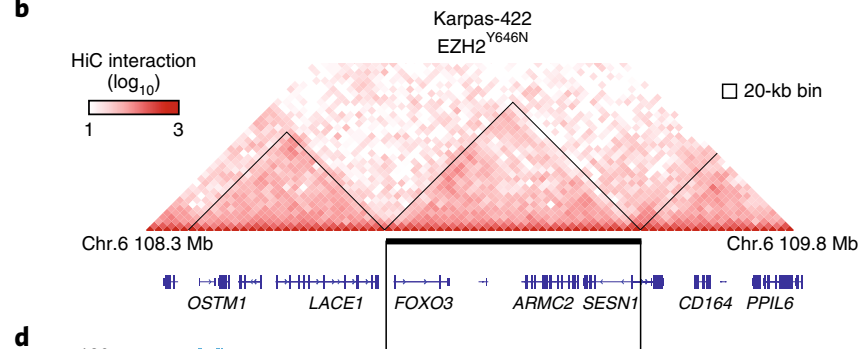

d

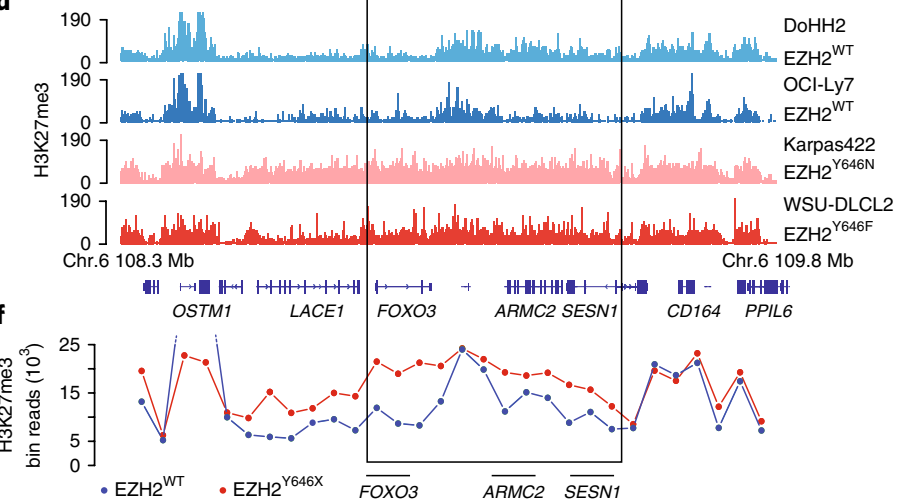

h
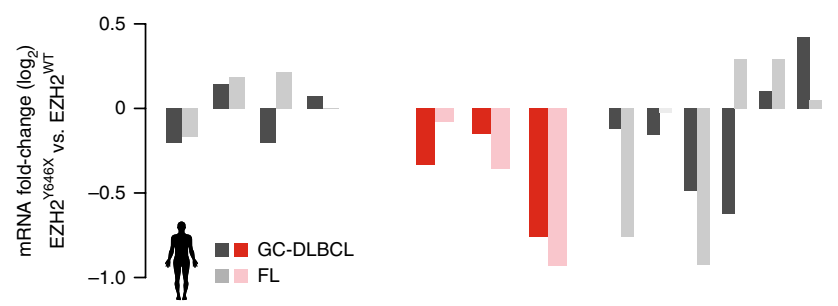

Fig. 3 | Inactivation of the tumor-suppressive TAD comprising FOXO3 and SESN1. a,b, Hi-C contact maps at 20-kb resolution of the genomic region Chr.6 108.3-109.8 Mb in OCl-Ly19-EZH2 ${ }^{\mathrm{WT}}$ (a) and Karpas422-EZH2 ${ }^{\mathrm{Y} 646 \mathrm{~N}}(\mathbf{b})$. TADs are contoured in black, and representative genes within the TADs are shown. c,d, H3K27me3 ChIP-seq tracks on Chr.6 108.3-109.8 Mb for OCI-Ly19-EZH2WT and OCI-Ly19-EZH2 ${ }^{\text {Y646F }}(n=3$ experiments per cell line) (c) and for the indicated $\mathrm{EZH} 2^{\mathrm{WT}}$ (DoHH2 and OCl-Ly7) and $\mathrm{EZH} 2^{\mathrm{Y} 646 \mathrm{X}}$ (Karpas422 and WSU-DLCL2) cell lines ( $n=2$ experiments per cell lines, except WSUDLCL2 for which $n=1$ ) (d). e,f, Superimposition of H3K27me3 number of reads within 50-kb bins in Chr.6 108.3-109.8 Mb for OCl-Ly19-EZH2WT and $\mathrm{OCl}-\mathrm{Ly} 19 \mathrm{EZH} 2^{\mathrm{Y} 646 \mathrm{~F}}$ (e) and EZH2 ${ }^{\mathrm{WT}}$ and $\mathrm{EZH} 2^{\mathrm{Y} 646 \mathrm{X}}$ (f) cells. $\mathbf{g}$, mRNA expression fold changes of genes in Chr.6 108.3-109.8 Mb obtained by comparing OCl-Ly19-EZH2 ${ }^{\mathrm{Y} 646 \mathrm{~F}}$ and OCl-Ly19-EZH2 ${ }^{\mathrm{WT}}$ cell lines and $\mathrm{EZH} 2^{\mathrm{Y} 646 \mathrm{X}}\left(n=12\right.$ samples) and EZH2 ${ }^{\mathrm{WT}}(n=4$ samples) cell lines. $\mathbf{h}$, mRNA expression fold changes of the genes in Chr.6 108.3-109.8 Mb obtained by comparing EZH2 ${ }^{\mathrm{Y} 646 \mathrm{X}}(n=7)$ versus EZH2 ${ }^{\mathrm{WT}}(n=30) \mathrm{GC}$-DLBCL patient samples and EZH2 ${ }^{\mathrm{Y} 646 \mathrm{X}}$ $(n=6)$ versus $\mathrm{EZH} 2^{\mathrm{WT}}(n=17) \mathrm{FL}$ patient samples. Fold changes corresponding to the inactive TAD are highlighted in red.

OCI-Ly19-EZH2 ${ }^{\text {WT }}$ cells. TAD expression fold changes were moderately yet significantly anticorrelated with TAD H3K27me3 fold changes (Pearson's correlation $=-0.31, \quad P=3.2 \times 10^{-21}$, Supplementary Fig. 3f). Moreover, TADs exhibiting an H3K27me3 fold changegreater than two and an mRNA expression fold changeless than negative two represented the majority (77\%) of TADs exhibiting at least a twofold difference in both molecular features (Supplementary Fig. 3f), a result consistent with EZH2 mutations increasing $\mathrm{H} 3 \mathrm{~K} 27 \mathrm{me} 3$ and repressing transcription. Within the cell-line cohort including Karpas422 and WSU-DLCL2 (GSE40792), we confirmed a significant anticorrelation between TAD mRNA and H3K27me3 fold changes (Pearson's correlation $=-0.23, P=6 \times 10^{-25}$, Fig. $2 \mathrm{k}$ ), and TADs exhibiting H3K27me3 fold changes greater than two and mRNA expression fold changes less than negative two represented $86 \%$ of the TADs exhibiting at least a twofold difference in both molecular features. This anticorrelation was even more pronounced when only TADs comprising the most differentially expressed genes were considered (Supplementary Fig. 3g,h). Overall, EZH2 ${ }^{\mathrm{Y} 646 \mathrm{X}}$-driven epigenetic and transcriptional changes within TADs were found to be significantly anticorrelated.

The interdependency between gene regulation and TAD structure prompted us to investigate TADs that exhibited a strong increase in $\mathrm{H} 3 \mathrm{~K} 27 \mathrm{me} 3$ and a strong decrease in gene expression. Within the full set of conserved TADs, we identified 72 'inactive' TADs characterized by agreater than twofold increase in $\mathrm{H} 3 \mathrm{~K} 27 \mathrm{me} 3$ and areater-than-twofold decrease in mRNA expression (Fig. 21). Notably, largely overlapping results were obtained when we considered OCI-Ly19 expression differences $\left(\mathrm{EZH} 2^{\mathrm{Y} 646 \mathrm{~F}}\right.$ versus EZH2 ${ }^{\text {WT }}$ ) or Karpas422 and WSU-DLCL2 H3K27me3 profiles (Supplementary Fig. 3i). Inactive TADs were enriched in genes that were significantly downregulated in $\mathrm{EZH} 2^{\mathrm{Y} 646 \mathrm{X}}$ cell lines (Fisher's exact test $P=2.8 \times 10^{-37}$, odds ratio $(\mathrm{OR})=12.4$ ). Moreover, in 32 of these 72 domains, more than $80 \%$ of the genes within the domain exhibited concordant negative fold changes. 
We defined these TADs as 'stringent inactive' (Fig. 2l) and the remaining 40 TADs as 'permissive inactive' (Fig. 2l). In primary patient samples, the mRNA fold changes in inactive TADs were significantly lower than those in other TADs (GC-DLBCL, two-tailed $t$ test $P=2.3 \times 10^{-5}$ and FL, two-tailed $t$ test $\left.P=1.7 \times 10^{-8}\right)$, and inactive TADs were enriched among TADs with concordant negative fold changes in more than $80 \%$ of the genes within their boundaries (Fisher's exact test: $P_{\mathrm{GC}-\mathrm{DLBCL}}=0.0006, \mathrm{OR}_{\mathrm{GC}-\mathrm{DLBCL}}=3.2$, and $\left.P_{\mathrm{FL}} 2.9 \times 10^{-5}, \mathrm{OR}_{\mathrm{FL}}=3.6\right)$.

To explore the functional relevance of inactive TADs, we performed a gene-set enrichment analysis on all genes within these domains. Multiple Gene Ontology categories scored as significant (Supplementary Table 3), including cancer and lymphoma-specific categories (Fig. 2m). Notably, most significantly enriched gene sets contained EZH2 targets previously identified in prostate cancer ${ }^{32}$ and genes differentially expressed in B-lymphocyte progenitors (Fig. $2 \mathrm{~m}$ and Supplementary Table 3 ). These findings are consistent with $\mathrm{EZH} 2^{\mathrm{Y} 646 \mathrm{X}}$ locking in an inactive state genes that are transiently repressed in the GC during B-cell differentiation ${ }^{21}$. To determine whether inactive TADs are also repressed in normal GC cells, we compared the mRNA expression of their genes in normal centroblasts and centrocytes versus differentiated memory B cells ${ }^{33}$. Across all 72 inactive TADs, we identified seven domains that exhibited significant downregulation in centrocytes and centroblasts compared with memory B cells (Supplementary Fig. 3j). Transcriptionally repressed domains included genes regulating B-cell proliferation (for example $S E S N 1)^{22}$, DNA repair (DTX3L and PARP9) $)^{34}$, and lymphocyte migration and trafficking (for example $S 1 P R 1)^{35}$ (Supplementary Fig. 3k).

Overall, these results suggest that genes within inactive TADs are bona fide targets of EZH2 and that inactivation of TADs containing genes involved in B-cell differentiation and proliferation is potentially selected in EZH2-mutated tumors.

Silencing multiple tumor suppressors in inactive TADs. One of the top stringent inactive TADs included the genes SESN1, FOXO3, and ARMC2 (TAD6.139 at chromosome (Chr.) 6 108850$109450 \mathrm{~kb}$; Fig. 3a,b). Recently, we reported that SESN1 is a direct target of mutated EZH2 and that it acts as a tumor suppressor in $\mathrm{FL}^{22}$. FOXO3 has been described as bona fide tumor-suppressor gene controlling multiple signalings ${ }^{36}$. Moreover, genes within this locus are frequently co-deleted in non-Hodgkin lymphoma, and these deletions are largely mutually exclusive with EZH2 mutations ${ }^{22,37,38}$. The inactive TAD6.139 exhibited a significant increase in H3K27me3 encompassing the entire domain in OCI-Ly19 cells overexpressing $\mathrm{EZH} 2^{\mathrm{Y} 646 \mathrm{~F}}$, whereas $\mathrm{H} 3 \mathrm{~K} 27 \mathrm{me} 3$ was detected only in specific regions in OCI-Ly19-EZH2 ${ }^{\text {WT }}$ cells (Fig. 3c,e). Similarly, an independent comparison of H3K27me3 levels in EZH2-mutated and wild-type cells confirmed a clear increase in $\mathrm{H} 3 \mathrm{~K} 27 \mathrm{me} 3$ within the entire inactive TAD (Fig. 3d,f). Consistently, all genes within TAD6.139 exhibited lower mRNA expression in OCI-Ly19-EZH2 ${ }^{\mathrm{Y} 646 \mathrm{~F}}$ and in EZH2-mutated lymphoma cells (Fig. 3g), as well as in GC-DLBCL and FL patient samples with EZH2 ${ }^{\mathrm{Y} 646 \mathrm{X}}$ mutations (Fig. 3h) compared with EZH2 ${ }^{\mathrm{WT}}$ lymphomas. Enrichment in H3K27me3 was confirmed by targeted ChIP (Supplementary Fig. 4a), and changes in expression in OCI-Ly19-EZH2 ${ }^{\mathrm{Y} 646 \mathrm{~F}}$ cells were confirmed by quantitative PCR (Supplementary Fig. 4b). Notably, additional genes with previously described tumor-suppressive function were found within inactive TADs (Supplementary Table 2). For example, similar patterns of $\mathrm{H} 3 \mathrm{~K} 27 \mathrm{me} 3$ and gene downregulation were confirmed in TAD3.47 (Chr.3 33150-33450 kb), encompassing the tumor-suppressor gene FBXL2 (refs. ${ }^{39,40}$ ) and silenced targets in lymphoma, $C R T A P^{41}$, and SUSD $5^{42}$ (Supplementary Fig. $4 c-e$ ). In particular, FBXL2 mediates degradation of cyclin-D3, which is the most expressed and mutated cyclin-D protein in B-cell lymphoma ${ }^{43}$.

Together, these results indicate that mutated EZH2 promotes concordant downregulation of multiple tumor suppressors within the same TAD, thus suggesting that these domains may function as tumor-suppressive units.

TAD inactivation accelerates lymphomagenesis. To assess the functional consequences of simultaneous downregulation of multiple tumor-suppressor genes within the same chromatin domain, we knocked down, alone and in combination, the expression of FOXO3, ARMC2, and SESN1, which are included in the candidate tumor-suppressive TAD6.139. We used IL3-dependent pro-B FL512 cells as a surrogate model to measure the effects of gene downregulation on B-cell proliferation ${ }^{38,44}$. We knocked down Foxo3, Sesn 1, and Armc2 by using short hairpin RNAs (shRNAs, denoted by 'sh'prefix) coupled with a fluorescent marker ${ }^{22,44}$ (Supplementary Fig. 5a-c). The number of cells expressing either shFoxo3 or shSesn 1 increased two to threefold after four cycles of IL3 withdrawal (12 days). Notably, in the same time frame, cells with the dual knockdown of Foxo3 and Sesn1 increased 7- to 12-fold (Fig. 4a,b, Supplementary Fig. 5d, and Supplementary Table 4). We observed a similar enrichment in double-positive cells in normal growth conditions (Supplementary Table 4), whereas a synergistic effect was not observed with concurrent knockdown of Armc2 and either Foxo3 or Sesn1 (Supplementary Fig. 5e,f), thus suggesting a specific functional synergy between loss of Foxo3 and Sesn 1 to enhance pro-B cell proliferation.

Next, to test the oncogenic potential of the dual loss of Foxo3 and Sesn 1 in tumor development and progression, we transduced hematopoietic progenitor cells (HPCs) isolated from an E $\mu$-myc mouse model ${ }^{45,46}$ with shRNAs targeting Foxo3, Sesn1, or both (Fig. 4c). Animals harboring tumors expressing shFoxo3 $(n=16)$, shSesn $1(n=17)$, or both $(n=21)$ had shorter overall survival than those expressing vector $(n=14$, shFoxo3 versus vector $P=0.04$, shSesn 1 versus vector $P=0.03$, and shFoxo3 and shSesn 1 versus vector $P=0.02$ ) (Supplementary Fig. $5 \mathrm{~g}$ ). Tumors that originated from HPCs transduced with single shRNAs were enriched in either

Fig. 4 | Concurrent downregulation of genes within tumor-suppressive TADs accelerates B-cell proliferation and lymphoma progression. a,

Representative flow cytometry analysis of FL5-12 cells expressing empty vectors coupled with dsRed or GFP, or the sh1-Foxo3-dsRed and the sh2-Sesn1GFP at days 0 and 12. Numbers indicate the percentages of cells in each subpopulation ( $n=3$ independent experiments). $\mathbf{b}$, Percentages of cells in each subpopulation at days 4,8 , and 12 , relative to day 0 . Bars indicate mean values, and error bars correspond to one standard deviation over three independent experiments. $P$ values were calculated by two-way analysis of variance (independent variables are shRNA and day of measurement). c, Study design of in vivo experiments: HPCs were isolated from E $\mu$-myc embryos, retrovirally modified with the indicated plasmids, and transplanted into recipient animals. $\mathbf{d}$, Representative flow cytometry analysis of HPC before transplantation (left column) and of isolated tumor cells (right column). Numbers indicate the percentages of cells in each subpopulation ( $\operatorname{sh} 2-\operatorname{Sesn} 1 n=3, \operatorname{sh} 1-$ Foxo $n n=4$, sh2-Sesn1+ sh1-Foxo $n n=4$, independent animals). e, Quantitative expression analysis of tumors expressing vector ( $n=4$ independent animals and two technical replicates), sh2-Sesn1 ( $n=5$ independent animals), sh1-Foxo3 ( $n=4$ independent animals), and sh2-Sesn1 and sh1-Foxo3 ( $n=6$ independent animals). Bars indicate mean values, and error bars correspond to one standard deviation. $P$ values were calculated with two-tailed $t$ test. f, Hematoxylin and eosin (H\&E) cellular staining and Ki-67 immunohistochemistry of tumor and liver tissue biopsies of $\mathrm{E} \mu$-myc mice expressing the indicated shRNAs or vector ( $n=2$ independent experiments). Scale bar, $200 \mu \mathrm{m}$.

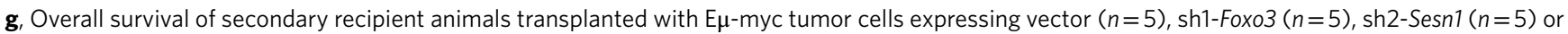
sh2-Sesn1 and sh1-Foxo3 $(n=5)$. $P$ value was calculated with log-rank test. 
GFP-positive (shSesn1) or dsRed-positive (shFoxo3) cells (Fig. 4d). Notably, even though HPCs transduced with both shRNAs showed a low percentage of double-positive cells in the initial population (5-8\%), tumors originating from these HPCs were almost exclusively composed of double-positive cells (Fig. 4d), thus suggesting that cells expressing both shRNAs expanded more rapidly than cells with a single shRNA. We confirmed that tumors expressing single or double shRNAs efficiently downregulated the expression of targeted genes (Fig. 4e), and all of the animals exhibited characteristic features of aggressive lymphoma (Fig. $4 \mathrm{f}$ and Supplementary Fig. 5h,i). To determine whether dual loss of Foxo3 and Sesn 1 boosts tumor aggressiveness, we transplanted purified tumor cells with single and double knockdown into secondary recipient animals. Here, we observed a significant acceleration of tumor development $(P=0.001)$ (Fig. $4 \mathrm{~g})$ in animals receiving cells with double knockdown compared with those with a single shRNA. These results a

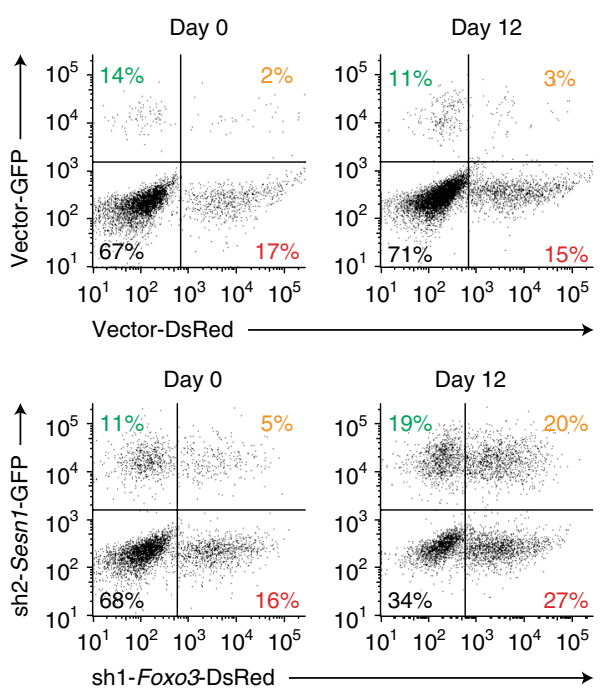

c

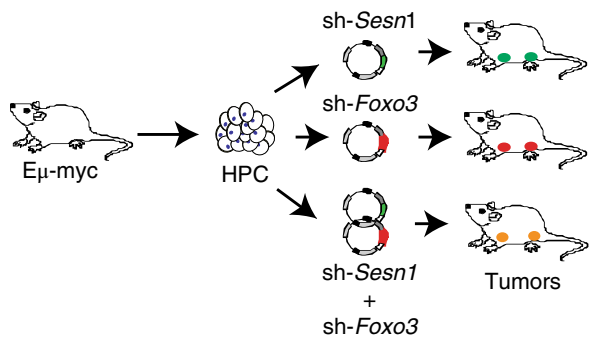

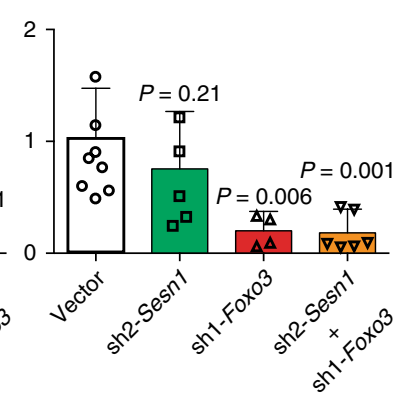

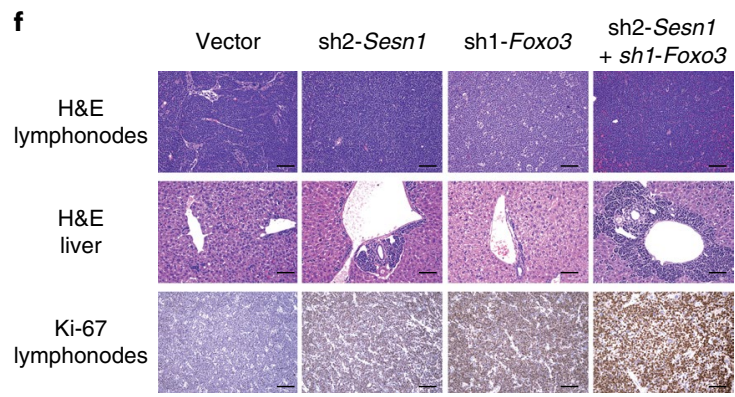

b
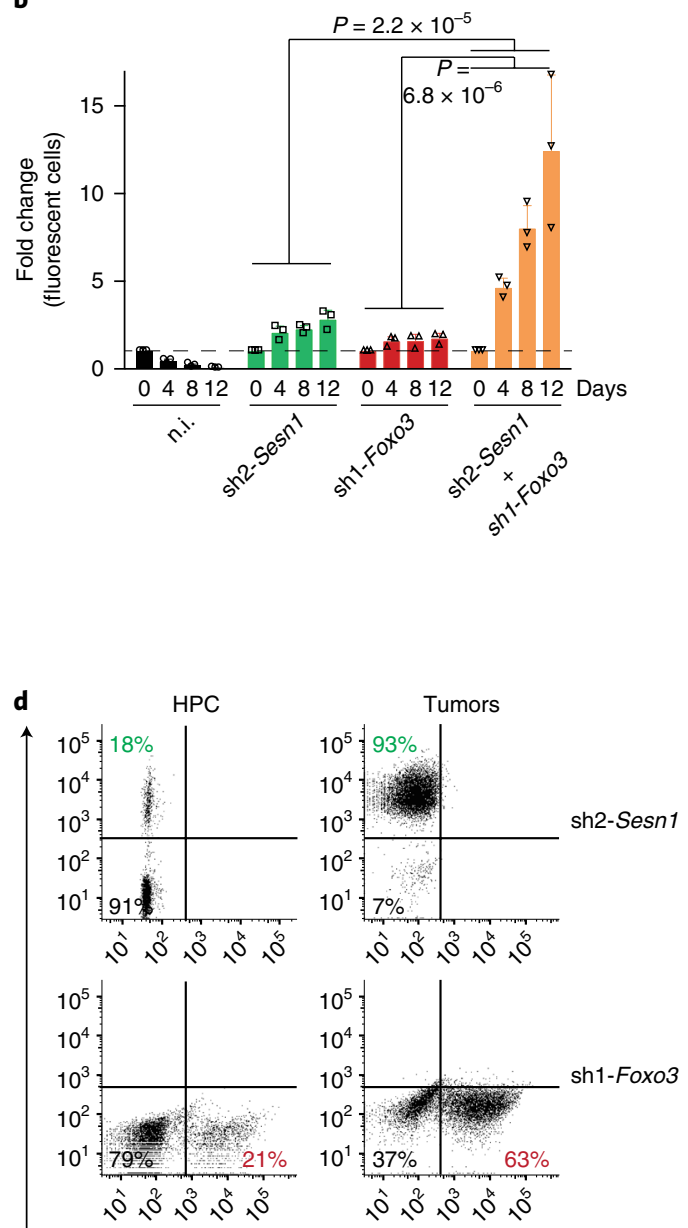

sh1-Foxo3
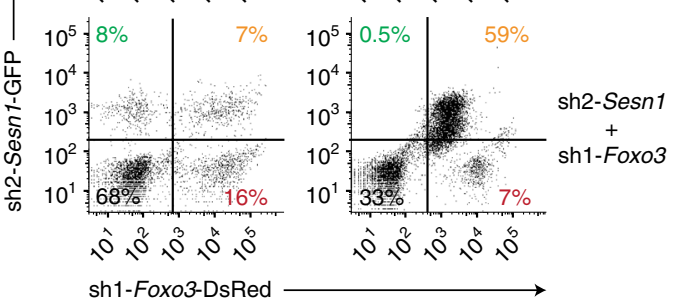

g

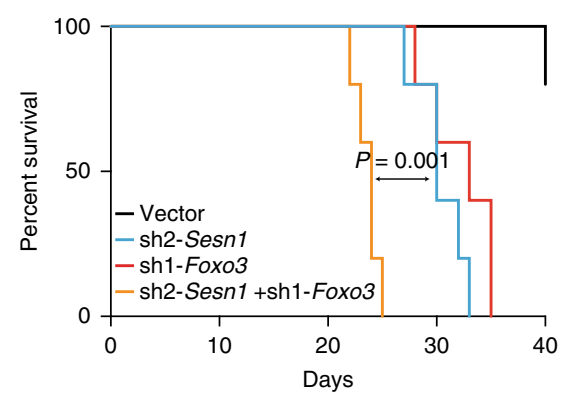




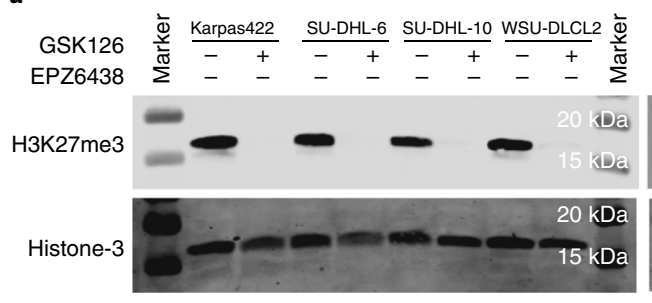

c

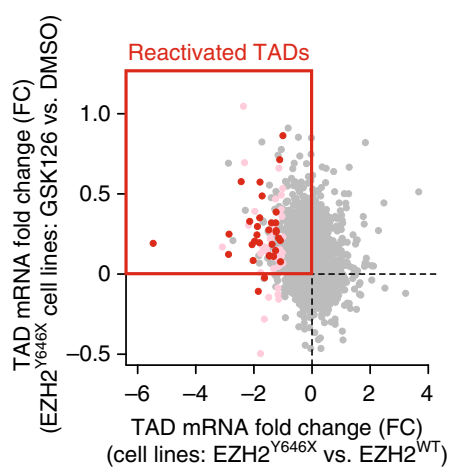

d

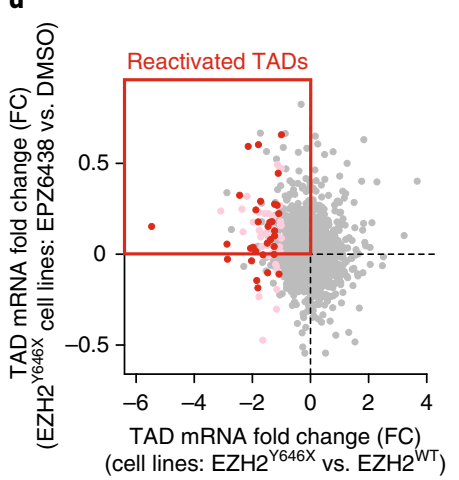

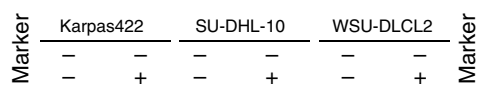

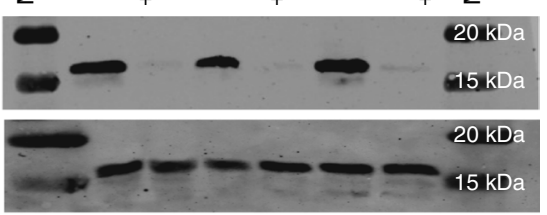

b

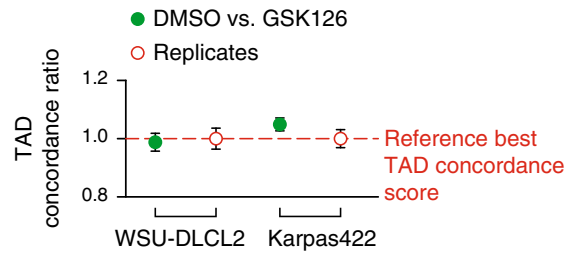

f Inactive TADs $\left[\begin{array}{l}\bullet \text { Stringent } \\ \text { Permissive }\end{array} \bullet\right.$ Other TADs Inactive TADs $\left[\begin{array}{l}\text { Stringent } \\ \text { Permissive }\end{array} \bullet\right.$ Other TADs Inactive TADs $\left[\begin{array}{l}\text { Stringent } \\ \text { Permissive }\end{array} *\right.$ Other TADs

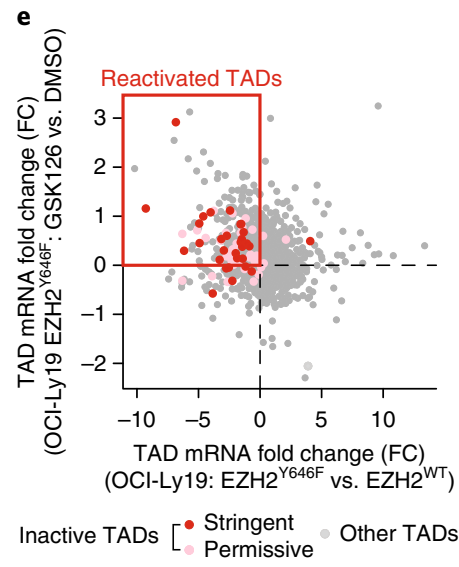

e

h

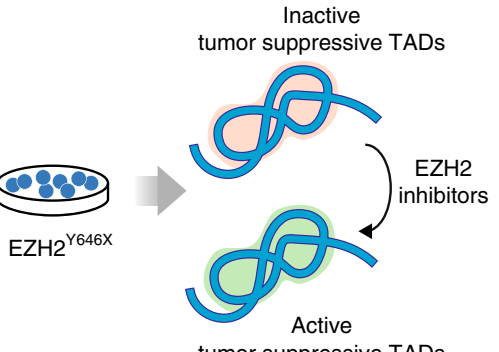

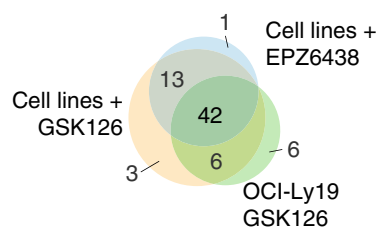

tumor suppressive TADs
Overlap reactivated TADs

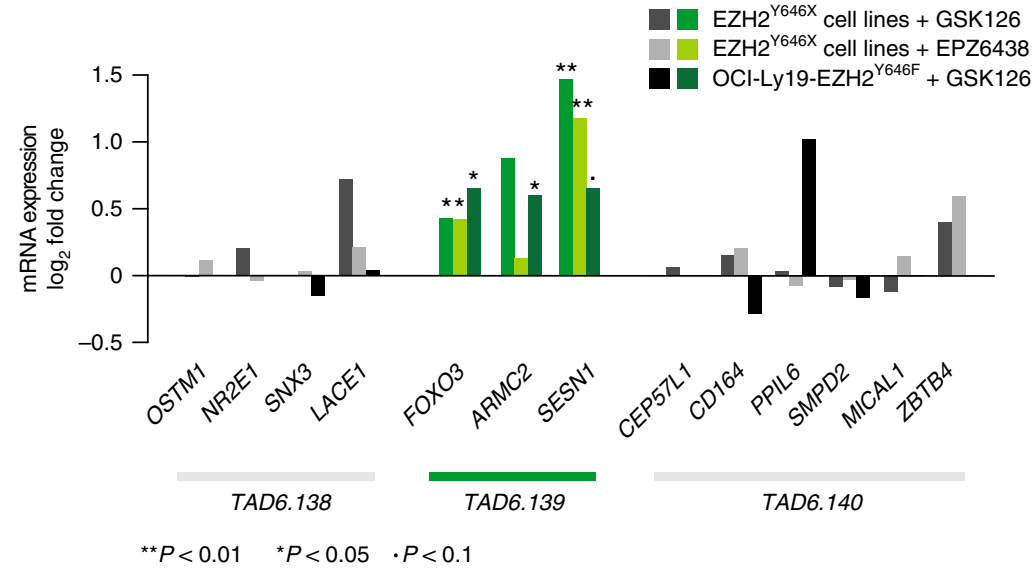

Fig. 5 | Inhibition of EZH2 abrogates H3K27me3 and reactivates inactive TADs. a, Immunoblot for $\mathrm{H} 3 \mathrm{~K} 27 \mathrm{me} 3$ in EZH2 ${ }^{\mathrm{Y} 646 \mathrm{X}}$ cell lines treated with $2 \mu \mathrm{M}$ of GSK126 or $1 \mu \mathrm{M}$ of EPZ6438 or vehicle (DMSO) for $72 \mathrm{~h}$. Total histone $\mathrm{H} 3$ was used as a loading control, and the experiment was repeated twice. b, Ratio of TAD concordance (MoC) between WSU-DLCL2 and Karpas422 treated with DMSO or GSK126 and TAD concordance between independent replicates of each cell line (DMSO). Dots indicate the means of 22 intrachromosomal-map comparisons, and error bars are \pm 1 standard deviation. c,d, TAD mRNA expression fold changes in EZH2 ${ }^{\mathrm{Y} 646 \mathrm{X}}(n=12)$ versus EZH2 ${ }^{\mathrm{WT}}(n=4)$ cell lines ( $x$ axis) versus TAD mRNA expression fold changes in $\mathrm{EZH} 2^{\mathrm{Y} 646 \mathrm{X}}$ cells treated with GSK126 $(n=12)$ versus DMSO $(n=12)(\mathbf{c})$ or treated with EPZ6438 ( $\left.n=12\right)$ versus DMSO ( $\left.n=12\right)(y$ axis) (d). e, TAD mRNA expression fold changes of OCl-Ly19-EZH2 ${ }^{\mathrm{Y} 646 \mathrm{~F}}$ versus OCl-Ly19-EZH2 ${ }^{\mathrm{WT}}$ ( $x$ axis) and in OCl-Ly19-EZH2 ${ }^{\mathrm{Y} 646 \mathrm{~F}}$ treated with GSK126 versus DMSO ( $y$ axis). f, Intersection of inactive TADs obtaining a $\log _{2}\left(\mathrm{FC}_{\mathrm{mRNA}}\right)>0$ (reactivated) in $\mathrm{EZH} 2^{\mathrm{Y} 646 \mathrm{X}}$ cell lines treated with GSK126 or EPZ6438, or OCI-Ly19-EZH2 ${ }^{\mathrm{Y} 646 \mathrm{~F}}$ cells treated with GSK126. g, mRNA expression fold changes of individual genes in Chr.6 108.3-109.8 Mb obtained by comparing EZH2 ${ }^{\mathrm{r} 646 \mathrm{X}}$ cell lines treated with GSK126 $(n=12)$ or EPZ6438 $(n=12)$ versus DMSO $(n=12)$ and OCl-Ly19-EZH2 ${ }^{\text {r646F }}$ treated with GSK126 $(n=3)$ versus vehicle $(n=3)$. Fold changes corresponding to the inactive TAD are highlighted in green; $P$ values were computed with limma two-sided $t$ test. $\mathbf{h}$, Graphical summary of the results: treatment with $\mathrm{EZH} 2$ inhibitors act as a switch turning tumor-suppressive TADs from transcriptionally inactive to active domains. ${ }^{\star} P<0.05$,

${ }^{\star} P<0.01, \bullet P<0.1$.

demonstrate that the inactivation of the tumor-suppressive TAD6.139 by mutated EZH2 synergistically downregulates multiple tumor suppressors to drive tumor development and progression.

EZH2 ${ }^{\mathrm{Y} 646 \mathrm{X}}$ inhibition reactivates transcription within TAD. Next, we explored the potential of blocking EZH2 methyltransferase activity by using two pharmacological inhibitors (GSK126 and EPZ6438) to reactivate inactive TADs. Pharmacological inhibition of EZH2 with either GSK126 or EPZ6438 efficiently depleted H3K27me3 in lymphoma cells (Fig. 5a). However, loss of H3K27me3 did not significantly modify the chromatin compartmentalization into TADs. Indeed, TADs derived from intrachromosomal maps of treated and untreated cells exhibited conservation scores similar to those observed for independent replicates of the same cell line (Fig. 5b). Nevertheless, EZH2 ${ }^{\mathrm{Y} 646 \mathrm{X}}$ cell lines treated with the EZH2 inhibitors GSK126 and EPZ6438 $(n=12$ treated and $n=12$ DMSO) exhibited multiple transcriptional changes, although not as extreme as those observed between $\mathrm{EZH} 2^{\mathrm{Y} 646 \mathrm{X}}$ and $\mathrm{EZH} 2^{\mathrm{WT}}$ 
cells (Supplementary Fig. 6a,b). By mapping differentially expressed genes to our list of conserved TADs, we found that the TAD mRNA expression changes induced by the two inhibitors were positively correlated (Supplementary Fig. 6c) and mostly corresponded to upregulation (positive fold changes) of TADs that were downregulated (negative fold changes) in $\mathrm{EZH} 2^{\mathrm{Y} 646 \mathrm{X}}$ compared with EZH2 ${ }^{\mathrm{WT}}$ cells (Supplementary Fig. 6d,e). Importantly, most inactive TADs (64/72 with GSK126 and 56/72 with EPZ6438) exhibited positive fold changes, and these reactivated TADs were enriched in significantly upregulated genes (adjusted $P$ value $<0.01$ ) from both experiments (Fig. 5c,d) (DMSO versus GSK126: Fisher's exact test $P=10^{-4}$, $\mathrm{OR}=2.74$, DMSO versus EPZ6438: Fisher's exact test $P=6.8 \times 10^{-8}$, $\mathrm{OR}=3.17)$. A similar reactivation $(54 / 72$ inactive TADs) was verified in OCI-Ly19-EZH2 ${ }^{\mathrm{Y} 646 \mathrm{~F}}$ cells treated with the GSK126 inhibitor (Fig. 5e and Supplementary Fig. 6f). Reactivated TADs had a high degree of overlap among the three experiments (Fig. 5f). In particular, after treatment with both EZH2 inhibitors, genes within the tumor-suppressive TAD6.139 exhibited positive fold changes in all three models, results consistent with a stringent inactive-toactive switch of the domain, in which all genes are concordantly regulated (Fig. 5g). Conversely, TADs flanking TAD6.139 showed no pattern of co-regulation. These results were validated through quantitative PCR (Supplementary Fig. 6g). Notably, treatment with EZH2 inhibitors increased FOXO3, SESN1, and ARMC2 expression exclusively in OCI-Ly19-EZH2 ${ }^{\mathrm{Y} 646 \mathrm{~F}}$ cells, whereas no significant expression changes were observed in the OCI-Ly19-EZH2 ${ }^{\text {WT }}$ cells (Supplementary Fig. 6h). Reactivation of the tumor-suppressive TAD6.139 is thus a direct effect of inhibiting the mutated form of EZH2. Together, these results indicate that pharmacological depletion of $\mathrm{H} 3 \mathrm{~K} 27 \mathrm{me} 3$ is sufficient to restore the transcriptional activity in previously silenced tumor-suppressive chromatin domains (Fig. 5h).

EZH2 ${ }^{\mathrm{Y} 646 \mathrm{X}}$ modulates intra-TAD chromatin interactions. Changes in transcriptional activity are frequently associated with novel or missing interactions among regulatory elements ${ }^{10,12,47}$. Although TADs in $\mathrm{EZH} 2^{\mathrm{Y} 646 \mathrm{X}}$, untreated or treated with $\mathrm{EZH} 2$ inhibitors, and EZH2 ${ }^{\text {WT }}$ cells were highly conserved (Supplementary Fig. 7a), we asked whether transcriptional changes in tumor-suppressive TADs were associated with rewiring of specific interactions within the domains.

To address this question within the TAD6.139, we compared 20-kb-resolution Hi-C maps for Karpas422 treated with vehicle (Karpas422-DMSO, Fig. 6a) and with GSK126 (Karpas422-GSK126, Fig. 6a). Significant interactions between the 5 ' ends of the domain (Chr.6 108860-108880 kb) and a region spanning $\sim 100 \mathrm{~kb}$ in the middle part of the TAD (Chr.6 109100-109220 kb) were detected in treated and untreated cells (Supplementary Table 5). However, only Karpas422 treated with GSK126 exhibited a highly significant interaction between genomic regions near the 5 ' and 3 ' ends of the domain (Chr.6 108860-108880 kb and 109380-109400 kb) (Fig. 6b). Next, we systematically compared significant interactions in Karpas422-DMSO and/or Karpas422-GSK126 to identify significant differences between these conditions (empirical $q$ value $<0.1$ ) (Fig. 6c). Notably, top-scoring differential interactions (empirical $q$ value $=0.025$ ) highlighted a stronger interaction between the $5^{\prime}$ end and the middle region of the domain in Karpas422-DMSO than Karpas422-GSK126, whereas Karpas422-GSK126 exhibited a significantly stronger interaction between the 5 ' and 3 ' ends of the domain than Karpas422-DMSO (Fig. 6c,d and Supplementary Table 5). This observation was confirmed by quantification of the number of normalized reads between the 20-kb regions Chr.6 108860-108880 kb and Chr.6 109380-109400 kb $(P=0.02)$ (Supplementary Fig. 7b), and the same significantly different interactions were found in WSU-DLCL2 cells treated with vehicle or GSK126 (Supplementary Fig. 7c-e). To corroborate the observed changes in interaction frequency at higher resolution, we performed UMI-4C in Karpas422, either untreated or treated with GSK126, by using two independent sets of primers (Supplementary Table 6). We observed an enrichment in interactions between the Chr.6 108861-108863kb and Chr.6 109370-109400 kb regions in cells treated with GSK126 compared with untreated cells (DMSO) with both primers (Fig. 6e,f and Supplementary Fig. 7f,g).

By mapping significantly differential interactions to functionally annotated regions in the genome, we found that these interactions linked genomic regions proximal to gene promoters that are recognizable by H3K4me3 peaks (Fig. $6 \mathrm{~g}$ and Supplementary Fig. 7h). Notably, chromatin contacts were stronger between genomic regions proximal to FOXO3 and SESN1 promoters in EZH2 ${ }^{\mathrm{Y} 646 \mathrm{X}}$ cells treated with GSK126 than in untreated cells (Fig. 6g). Concordant with the inactive status of TAD6.139 in $\mathrm{EZH} 2^{\mathrm{Y} 646 \mathrm{X}}$ cells, the promoters of FOXO3, ARMC2, and SESN1 exhibited lower H3K4me3 and higher $\mathrm{H} 3 \mathrm{~K} 27 \mathrm{me} 3$ in $\mathrm{EZH} 2^{\mathrm{Y} 646 \mathrm{X}}$ than in $\mathrm{EZH} 2^{\mathrm{WT}}$ cells (Fig. $6 \mathrm{~g}$ and Supplementary Fig. 7h). Moreover, a peak of H3K27ac and H3K4me1 was detected at the SESN1 promoter only in OCI-Ly7 $\mathrm{EZH} 2^{\mathrm{WT}}$ cells, thus suggesting that in these cells, this promoter might have distal enhancer function ${ }^{48}$ (Supplementary Fig. 7h).

To determine whether treatment with EZH2 inhibitor restored intra-TAD interactions that were modified by the increase of H3K27me3 in EZH2 mutated tumors, we performed the same differential-interaction comparison between syngeneic $\mathrm{EZH} 2^{\mathrm{Y} 646 \mathrm{~F}}$ and EZH2 ${ }^{\text {WT }}$ OCI-Ly19 cells as well as between Karpas422 (EZH2 $\left.{ }^{\mathrm{Y} 646 \mathrm{~N}}\right)$ and OCI-Ly19 $\left(\mathrm{EZH} 2^{\mathrm{WT}}\right)$. OCI-Ly19-EZH2 ${ }^{\mathrm{WT}}$ showed stronger interactions between FOXO3 and SESN1 promoter regions than OCI-Ly19 cells expressing EZH2 ${ }^{\mathrm{Y} 646 \mathrm{~F}}$ (Fig. 6h). The same difference in interaction between these loci was detected between OCILy19-EZH2 ${ }^{\text {WT }}$ and Karpas422-EZH2 ${ }^{\mathrm{Y} 646 \mathrm{~N}}$ (Supplementary Fig. 7i). Conversely, both OCI-Ly19-EZH2 ${ }^{\mathrm{Y} 646 \mathrm{~F}}$ and Karpas422-EZH2 $2^{\mathrm{Y} 646 \mathrm{~N}}$

Fig. 6 | Intra-TAD structural changes in EZH2 ${ }^{\mathrm{wT}}$ and EZH2 ${ }^{\mathrm{Y} 646 \mathrm{x}}$ cells. a, $\mathrm{Hi}-\mathrm{C}$ contact maps of TAD6.139 at 20-kb resolution (Chr.6 108.84-109.44 Mb) in Karpas422 treated with DMSO (top) or $2 \mu$ M GSK126 for $72 \mathrm{~h}$ (bottom). b. Significant interactions in Karpas422-DMSO (top) or Karpas422-GSK126 (bottom) determined by HiC-DC. Bin pairs within 2-Mb windows were tested. c, Significantly different interactions $(q$ value $<0.1)$ between Karpas422DMSO and Karpas422-GSK126 ( $n=435$ tested interactions). Empirical $q$ values were determined as described in Methods. $\mathbf{d}$, Most significantly different interactions between Karpas422-DMSO and Karpas422-GSK126. e, UMI-4C domainogram: mean number of contacts (percentage of the maximum) in the Chr.6 108.80-109.45 kb region. f, Normalized number of UMI-4C reads in Karpas422-DMSO and Karpas422-GSK126. g, Significantly stronger interaction (compare to c) in Karpas422-GSK126 than Karpas422-DMSO connecting Chr.6 108.85-108.81 Mb and Chr.6 109.39-109.45 Mb. H3K4me3 and H3K27me3 ChIP-seq tracks in Karpas422 and OCI-Ly7 ( $n=3$ experiments) within the same genomic coordinates. h, Left, Hi-C contact maps of TAD6.139 at 20-kb resolution in OCl-Ly19-EZH2 ${ }^{\mathrm{Y} 646 \mathrm{~F}}$ (top) and OCl-Ly19-EZH2 ${ }^{\mathrm{WT}}$ (bottom). Center, significant interactions in OCl-Ly19-EZH2 ${ }^{\mathrm{Y} 646 \mathrm{~F}}$ (top) or OCl-Ly19EZH2 ${ }^{\text {WT }}$ (bottom) determined by HiC-DC. Top right, significantly different interactions ( $q$ value $<0.1$ ) between OCI-Ly19-EZH2 ${ }^{\mathrm{Y} 646 \mathrm{~F}}$ and OCl-Ly19-EZH2WT ( $n=435$ tested interactions). Empirical $q$ values were determined as described in Methods. Bottom right, most significantly different interactions between OCl-Ly19-EZH2 ${ }^{\mathrm{Y} 646 \mathrm{~F}}$ and OCI-Ly19-EZH2 ${ }^{\mathrm{WT}}$. i, FISH library design for TAD6.139 and representative images acquired in wide field (left) and by STORM (right). j, Eccentricity of TAD6.139 (neighborhood radius $=40$ ) in WSU-DLCL2 treated with $2 \mu \mathrm{M} \mathrm{GSK126}$ or DMSO for $72 \mathrm{~h}(n=4$ experiments), and OCILy19-EZH2 ${ }^{\mathrm{WT}}$ and OCl-Ly19-EZH2 ${ }^{\mathrm{Y} 646 \mathrm{~F}}$ ( $n=3$ experiments). Dots are means of multiple measurements of independent cells (Supplementary Table 7), and error bars are standard errors. 
exhibited stronger interactions between promoter regions of $\mathrm{FOXO} 3$ and ARMC2 than OCI-Ly19-EZH2 ${ }^{\mathrm{WT}}$ (Fig. 6h and Supplementary Fig. 7i). To verify whether changes in promoter proximity also occurred in other inactive TADs, we performed a differential interactome analysis within the complete set of 72 inactive TADs, as well as within 100 sets of 72 randomly sampled neutral TADs. By comparing Karpas422-EZH2 ${ }^{\mathrm{Y} 646 \mathrm{~N}}$ and OCI-Ly19-EZH2 ${ }^{\mathrm{WT}}$ cells, we found 34 interactions that were significantly different within inactive TADs, but only 19 on average in neutral TADs $(P=0.04$, Supplementary Fig. 7j). Furthermore, significant different interactions between $\mathrm{EZH} 2^{\mathrm{WT}}$ and $\mathrm{EZH} 2^{\mathrm{Y} 646 \mathrm{X}}$ cells were highly overlapping with those observed between cells treated with the EZH2 inhibitor GSK126 and untreated cells (17 out of 34, Fisher's exact test $P=6 \times 10^{-20}, \mathrm{OR}=130$ ) (Supplementary Fig. $7 \mathrm{k}$ ). In summary, we a

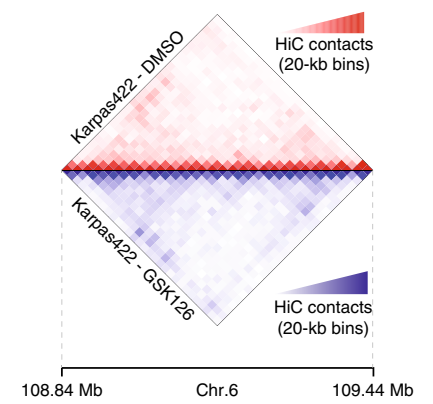

b

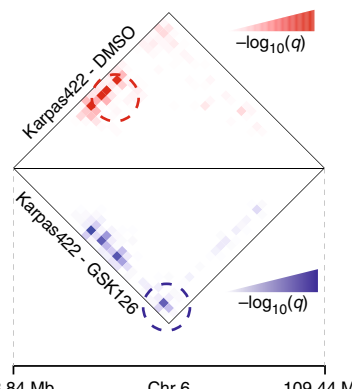

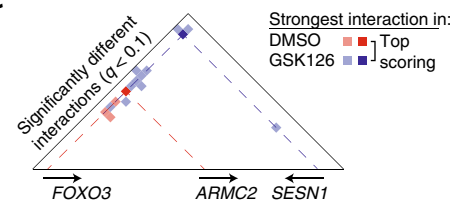

d

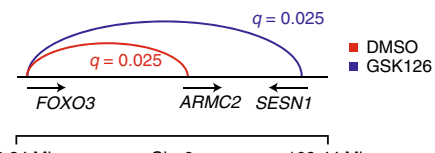

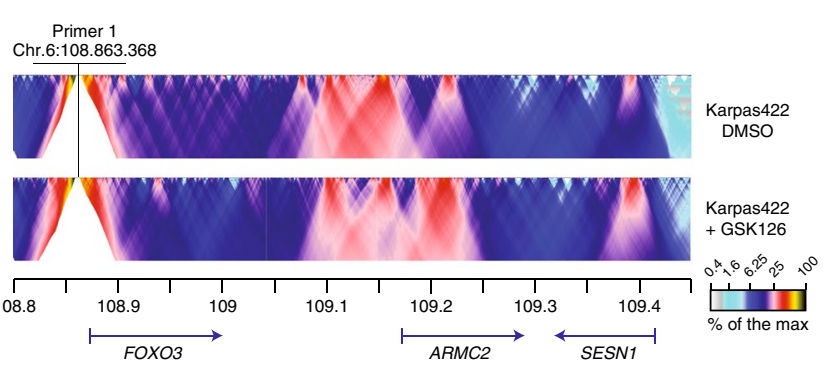

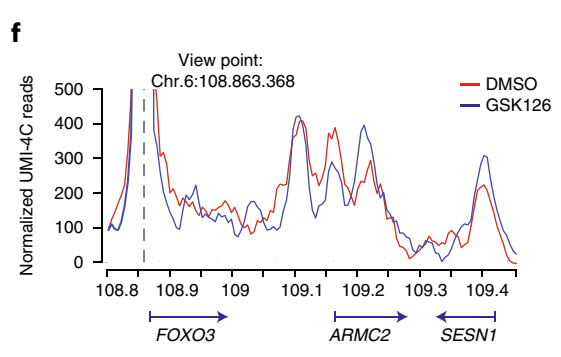

g

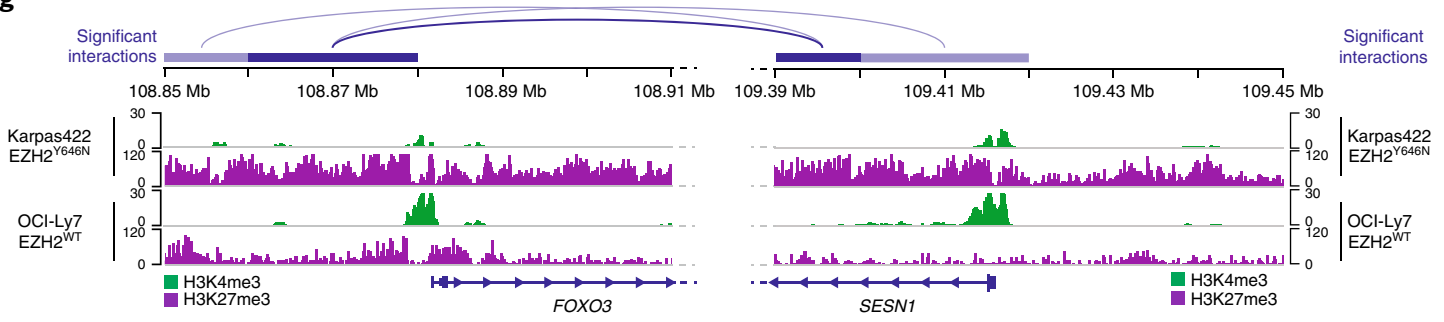

h
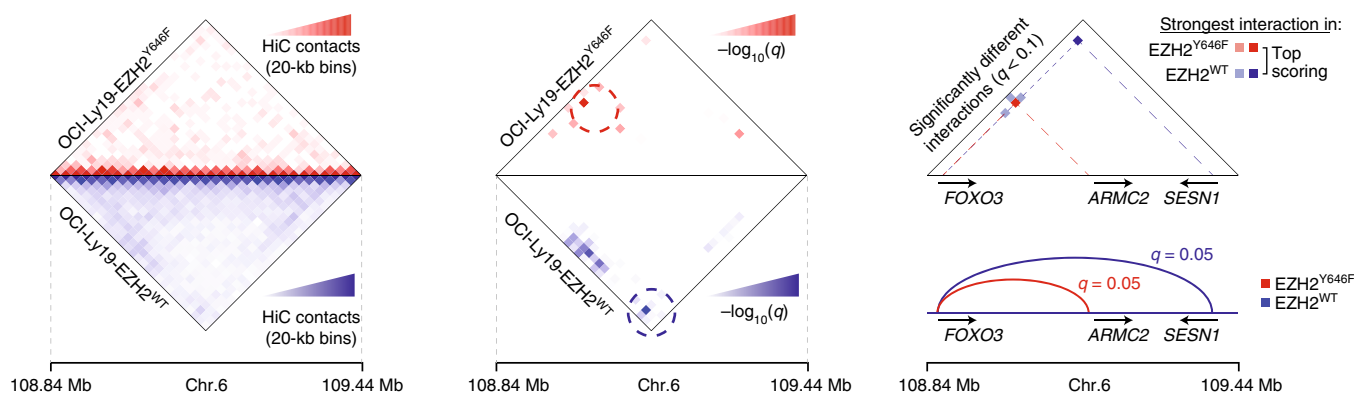

i

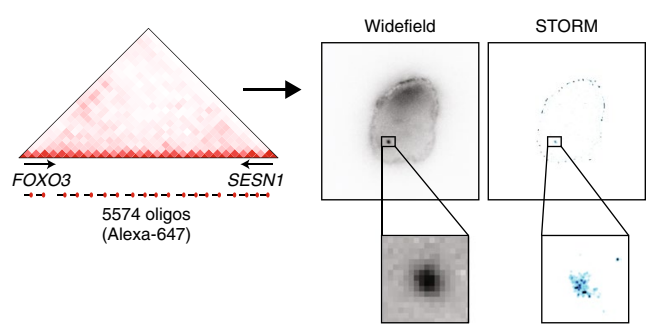

j

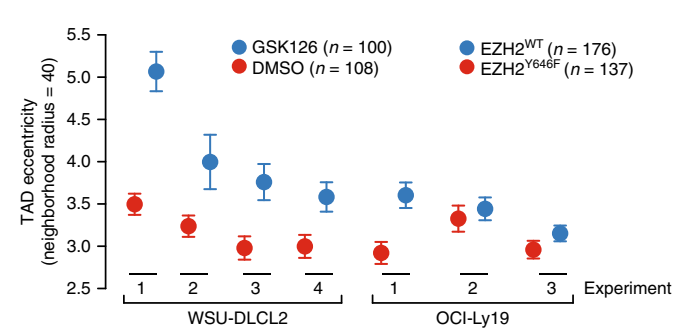


confirmed in multiple conditions, that the increase in $\mathrm{H} 3 \mathrm{~K} 27 \mathrm{me} 3$ in EZH2-mutated tumors modulates promoter-promoter interactions within inactive TADs, which are coupled with silencing of gene transcription.

To investigate the degree of observable physical changes of the intra-TAD structure, we used a super-resolution imaging technique (stochastic optical reconstruction microscopy, or STORM) $)^{49,50}$ and analyzed WSU-DLCL2 (DMSO versus GSK126, $n=4$ experiments) and OCI-Ly19 cells (EZH2 $2^{\mathrm{Y} 646 \mathrm{~F}}$ versus EZH2 ${ }^{\mathrm{WT}}, n=3$ experiments). To image the TAD structure by STORM, we designed a fluorescence in situ hybridization (FISH) library painting the entire TAD6.139 (Fig. 6i, Supplementary Fig. 8a, and Supplementary Note). TAD6.139 did not change significantly in size (as estimated by radius of gyration, $R_{\mathrm{g}}$ ) between the two conditions in all experiments (Supplementary Table 7). However, we observed consistent changes in shape, as evidenced by higher eccentricity values in $\mathrm{EZH} 2{ }^{\mathrm{WT}}$ and EZH $2{ }^{\mathrm{Y} 646 \mathrm{X}}$-GSK126 cells than in EZH2 ${ }^{\mathrm{Y} 646 \mathrm{X}}$ cells (Fig. $6 \mathrm{j}$ and Supplementary Fig. 8b-e). This increase in eccentricity was not observed in a neutral TAD of similar size (TAD1.54, Chr.1: 28650 $29100 \mathrm{~kb}$ ), exhibiting neither transcriptional nor epigenetic changes (Supplementary Fig. 8f,g).

Overall, our results indicate that the increase in $\mathrm{H} 3 \mathrm{~K} 27 \mathrm{me} 3$ associated with $E Z H 2$ gain-of-function mutations can modify the architecture and transcriptional activity within TADs.

\section{Discussion}

The recent advent of chromatin-conformation-capture techniques provides new opportunities and perspectives to unravel the effects of chromatin remodeling in cancer. EZH2 ${ }^{\mathrm{Y} 646 \mathrm{X}}$ increases $\mathrm{H} 3 \mathrm{~K} 27 \mathrm{me} 3$ across the genome, thus leading to transcriptional repression. Here, we found that these epigenetic and transcriptional changes were not randomly distributed but were enriched and concordant within specific TADs, termed inactive TADs. Although future work will be needed to explore whether this specificity is predetermined or emerges through selection of oncogenic traits, inactive TADs were enriched in both EZH2 targets and B-cell differentiation programs, thus suggesting that both scenarios are likely and play a role in implementing $\mathrm{EZH} 2^{\mathrm{Y} 646 \mathrm{X}}$ oncogenic potential.

$\mathrm{H} 3 \mathrm{~K} 27 \mathrm{me} 3$ determines changes in nucleosome proximity and is a marker of closed chromatin. Using multiple models, we showed that $\mathrm{H} 3 \mathrm{~K} 27 \mathrm{me} 3$ modulates the proximity of gene promoters in association with gene expression changes. In particular, concordant expression changes of FOXO3 and SESN1 suggest co-regulated transcription. Interestingly, both genes are direct transcriptional targets of p53 (refs. ${ }^{51,52}$ ), thus prompting the hypothesis that promoter proximity may favor coordinated transcription of genes controlled by the same transcription factor.

Coordinated epigenetic and transcriptional reprogramming within TADs led to the synergistic inactivation of multiple tumor suppressors, enhancing B-cell proliferation and tumor growth. On the basis of these results, we introduced the concept of tumorsuppressive TADs. Importantly, analysis of expression changes in individual genes, rather than TADs, would probably have missed this synergistic effect. Moreover, although relatively small mRNA expression changes for individual genes are typically dismissed as random variations, the unexpected concordance of these variations within TADs could identify functionally relevant gene modules.

Chromatin domains can therefore provide a new lens through which to study oncogenic alterations, especially in the context of cancer epigenetic reprogramming.

URLs. ENCODE, https://www.encodeproject.org/; GEO, https:// www.ncbi.nlm.nih.gov/geo/; GitHub custom scripts, https://github. com/CSOgroup/Donaldson-et-al-scripts/; Zenodo, https://zenodo. org/; NCBI BioProject https://www.ncbi.nlm.nih.gov/bioproject/; Bioconductor, https://bioconductor.org/; R, https://www.r-project. org/; Cufflinks, http://cole-trapnell-lab.github.io/cufflinks/; STAR, https://github.com/alexdobin/STAR/; Bowtie, http://bowtie-bio. sourceforge.net/bowtie2/manual.shtml; MACS2, https:/github. com/taoliu/MACS/; Picard, http://broadinstitute.github.io/picard/; LIMMA, http://bioconductor.org/packages/release/bioc/html/ limma.html; Bedtools, https://bedtools.readthedocs.io/en/latest/; HiC-DC, https://bitbucket.org/leslielab/hic-dc/; TopDom, http:// zhoulab.usc.edu/TopDom/; mSIGdb, http://software.broadinstitute. org/gsea/msigdb/index.jsp; hg19 mappability file, http://hgdownload.soe.ucsc.edu/goldenPath/hg19/encodeDCC/wgEncodeMapability/wgEncodeCrgMapabilityAlign36mer.bigWig.

\section{Online content}

Any methods, additional references, Nature Research reporting summaries, source data, statements of data availability and associated accession codes are available at https://doi.org/10.1038/ s41588-018-0338-y.

Received: 27 April 2018; Accepted: 17 December 2018; Published online: 28 January 2019

\section{References}

1. Bonev, B. \& Cavalli, G. Organization and function of the 3D genome. Nat. Rev. Genet. 17, 661-678 (2016).

2. Dixon, J. R., Gorkin, D. U. \& Ren, B. Chromatin domains: the unit of chromosome organization. Mol. Cell 62, 668-680 (2016).

3. Lieberman-Aiden, E. et al. Comprehensive mapping of long-range interactions reveals folding principles of the human genome. Science 326, 289-293 (2009)

4. Dixon, J. R. et al. Topological domains in mammalian genomes identified by analysis of chromatin interactions. Nature 485, 376-380 (2012).

5. Sexton, T. et al. Three-dimensional folding and functional organization principles of the Drosophila genome. Cell 148, 458-472 (2012).

6. Nora, E. P. et al. Spatial partitioning of the regulatory landscape of the X-inactivation centre. Nature 485, 381-385 (2012).

7. Le Dily, F. et al. Distinct structural transitions of chromatin topological domains correlate with coordinated hormone-induced gene regulation. Genes Dev. 28, 2151-2162 (2014)

8. de Laat, W. \& Duboule, D. Topology of mammalian developmental enhancers and their regulatory landscapes. Nature 502, 499-506 (2013).

9. Fraser, J. et al. Hierarchical folding and reorganization of chromosomes are linked to transcriptional changes in cellular differentiation. Mol. Syst. Biol. 11, 852 (2015).

10. Hnisz, D. et al. Activation of proto-oncogenes by disruption of chromosome neighborhoods.Science 351, 1454-1458 (2016).

11. Weischenfeldt, J. et al. Pan-cancer analysis of somatic copy-number alterations implicates IRS4 and IGF2 in enhancer hijacking. Nat. Genet. 49, 65-74 (2017).

12. Flavahan, W. A. et al. Insulator dysfunction and oncogene activation in IDH mutant gliomas. Nature 529, 110-114 (2016).

13. Taberlay, P. C. et al. Three-dimensional disorganisation of the cancer genome occurs coincident with long range genetic and epigenetic alterations. Genome Res. 26, 719-731 (2016).

14. Plass, C. et al. Mutations in regulators of the epigenome and their connections to global chromatin patterns in cancer. Nat. Rev. Genet. 14, 765-780 (2013)

15. Morin, R. D. et al. Somatic mutations altering EZH2 (Tyr641) in follicular and diffuse large B-cell lymphomas of germinal-center origin. Nat. Genet. 42, 181-185 (2010).

16. Souroullas, G. P. et al. An oncogenic Ezh2 mutation induces tumors through global redistribution of histone 3 lysine 27 trimethylation. Nat. Med. 22, 632-640 (2016).

17. Tirode, F. et al. Genomic landscape of Ewing sarcoma defines an aggressive subtype with co-association of STAG2 and TP53 mutations. Cancer Discov. 4, 1342-1353 (2014).

18. Comet, I., Riising, E. M., Leblanc, B. \& Helin, K. Maintaining cell identity: PRC2-mediated regulation of transcription and cancer. Nat. Rev. Cancer 16, 803-810 (2016)

19. Sneeringer, C. J. et al. Coordinated activities of wild-type plus mutant EZH2 drive tumor-associated hypertrimethylation of lysine 27 on histone $\mathrm{H} 3$ (H3K27) in human B-cell lymphomas. Proc. Natl Acad. Sci. USA 107, 20980-20985 (2010).

20. Yap, D. B. et al. Somatic mutations at EZH2 Y641 act dominantly through a mechanism of selectively altered PRC2 catalytic activity, to increase H3K27 trimethylation. Blood 117, 2451-2459 (2011). 
21. Béguelin, W. et al. EZH2 is required for germinal center formation and somatic EZH2 mutations promote lymphoid transformation. Cancer Cell 23, 677-692 (2013).

22. Oricchio, E. et al. Genetic and epigenetic inactivation of SESTRIN1 controls mTORC1 and response toEZH2 inhibition in follicular lymphoma. Sci. Transl. Med. 9, eaak9969 (2017).

23. Rao, S. S. P. et al. A 3D map of the human genome at kilobase resolution reveals principles of chromatin looping. Cell 159, 1665-1680 (2014).

24. Yang, T. et al. HiCRep: assessing the reproducibility of Hi-C data using a stratum-adjusted correlation coefficient. Genome Res. 27, 1939-1949 (2017)

25. Pfitzner, D., Leibbrandt, R. \& Powers, D. Characterization and evaluation of similarity measures for pairs of clusterings. Knowl. Inf. Syst. 19, 361 (2009).

26. Shin, H. et al. TopDom: an efficient and deterministic method for identifying topological domains in genomes. Nucleic Acids Res. 44, e70 (2016).

27. Zufferey, M., Tavernari, D., Oricchio, E. \& Ciriello, G. Comparison of computational methods for the identification of topologically associating domains. Genome. Biol. 19, 217 (2018).

28. Carty, M. et al. An integrated model for detecting significant chromatin interactions from high-resolution Hi-C data. Nat. Commun. 8, 15454 (2017).

29. Jin, F. et al. A high-resolution map of the three-dimensional chromatin interactome in human cells. Nature 503, 290-294 (2013).

30. McCabe, M. T. et al. EZH2 inhibition as a therapeutic strategy for lymphoma with EZH2-activating mutations. Nature 492, 108-112 (2012).

31. Ortega-Molina, A. et al. The histone lysine methyltransferase KMT2D sustains a gene expression program that represses B cell lymphoma development. Nat. Med. 21, 1199-1208 (2015).

32. Nuytten, M. et al. The transcriptional repressor NIPP1 is an essential player in EZH2-mediated gene silencing. Oncogene 27, 1449-1460 (2008).

33. Klein, U. et al. Transcriptional analysis of the B cell germinal center reaction. Proc. Natl Acad. Sci. 100, 2639-2644 (2003).

34. Yang, C.-S. et al. Ubiquitin modification by the E3 ligase/ADPribosyltransferase Dtx3L/Parp9. Mol. Cell 66, 503-516.e5 (2017).

35. Matloubian, M. et al. Lymphocyte egress from thymus and peripheral lymphoid organs is dependent on S1P receptor 1. Nature 427, 355-360 (2004).

36. Dansen, T. B. \& Burgering, B. M. T. Unravelling the tumor-suppressive functions of FOXO proteins. Trends. Cell Biol. 18, 421-429 (2008).

37. Okosun, J. et al. Integrated genomic analysis identifies recurrent mutations and evolution patterns driving the initiation and progression of follicular lymphoma. Nat. Genet. 46, 176-181 (2014).

38. Oricchio, E. et al. The Eph-receptor A7 is a soluble tumor suppressor for follicular lymphoma. Cell 147, 554-564 (2011).

39. Chen, B. B., Glasser, J. R., Coon, T. A. \& Mallampalli, R. K. F-box protein FBXL2 exerts human lung tumor suppressor-like activity by ubiquitinmediated degradation of cyclin D3 resulting in cell cycle arrest. Oncogene 31, 2566-2579 (2012)

40. Li, L., Pan, D., Chen, H., Zhang, L. \& Xie, W. F-box protein FBXL2 inhibits gastric cancer proliferation by ubiquitin-mediated degradation of forkhead box M1. FEBS Lett. 590, 445-452 (2016).

41. Hatzimichael, E. et al. The collagen prolyl hydroxylases are novel transcriptionally silenced genes in lymphoma. Br. J. Cancer 107, 1423-1432 (2012).

42. Chambwe, N. et al. Variability in DNA methylation defines novel epigenetic subgroups of DLBCL associated with different clinical outcomes. Blood 123 1699-1708 (2014)

43. Oricchio, E. et al. Frequent disruption of the RB pathway in indolent follicular lymphoma suggests a new combination therapy. J. Exp. Med. 211, 1379-1391 (2014).

44. Mavrakis, K. J. et al. Genome-wide RNA-mediated interference screen identifies miR-19 targets in Notch-induced T-cell acute lymphoblastic leukaemia. Nat. Cell Biol. 12, 372-379 (2010)
45. Scuoppo, C. et al. A tumour suppressor network relying on the polyaminehypusine axis. Nature 487, 244-248 (2012).

46. Schatz, J. H. et al. Targeting cap-dependent translation blocks converging survival signals by AKT and PIM kinases in lymphoma. J. Exp. Med. 208, 1799-1807 (2011).

47. Lupiáñez, D. G. et al. Disruptions of topological chromatin domains cause pathogenic rewiring of gene-enhancer interactions. Cell 161, 1012-1025 (2015).

48. Dao, L. T. M. et al. Genome-wide characterization of mammalian promoters with distal enhancer functions. Nat. Genet. 49, 1073-1081 (2017).

49. Boettiger, A. N. et al. Super-resolution imaging reveals distinct chromatin folding for different epigenetic states. Nature 529, 418-422 (2016).

50. Rust, M. J., Bates, M. \& Zhuang, X. Sub-diffraction-limit imaging by stochastic optical reconstruction microscopy (STORM). Nat. Methods 3, 793-796 (2006).

51. Budanov, A. V. \& Karin, M. The p53-regulated Sestrin gene products inhibit mTOR signaling. Cell 134, 451-460 (2008).

52. Renault, V. M. et al. The pro-longevity gene FoxO3 is a direct target of the p53 tumor suppressor. Oncogene 30, 3207-3221 (2011).

\section{Acknowledgements}

We thank B. Ren and A. D. Schmitt for help with Hi-C library preparation; C. Bolt for help with the UMI-4C protocol; J. Lingner, D. Trono, and F. Radtke for critical reading of the manuscript; and D. Duboule and J. Huelsken for useful discussions. We thank the EPFL research animal, flow cytometry, histology, and sequencing facilities. This work is supported by the ISREC Foundation (E.O.), the Swiss National Science Foundation (E.O. and M.C.D.-C. SNF-31003A_159637) and Swiss Cancer League (E.O. KFS-3982-082016). G.C. is supported by the Giorgi-Cavaglieri Foundation. D.T. is supported by the Swiss National Science Foundation (SNSF, SNF-310030_169519), M.Z. is supported by the Swiss Cancer League (KFS-3983-08-2016), and S.S. is supported by European Union's Horizon 2020 research and innovation program under the Marie Skłodowska-Curie grant agreement no. 665667 .

\section{Author contributions}

M.C.D-C. prepared Hi-C, UMI-4C, ChIP-seq and RNA-seq libraries and performed in vitro validation experiments and DNA FISH experiments. S.S. analyzed Hi-C, UMI-4C, and ChIP-seq data. M.Z. performed the comparison of Hi-C contact maps, TAD calling, and all analyses based on mRNA expression data. D.T. performed interactome analyses and all analyses based on ChIP-seq data. K.M.D. and T.R. acquired and analyzed STORM images. N.K. and E.B. performed in vitro and in vivo experiments. M.M. assisted in the analysis of Hi-C data. F.R. assisted in the analysis of STORM data. S.M. supervised in STORM acquisition and image analyses. G.C and E.O. designed the study and wrote the manuscript with comments from all authors.

\section{Competing interests}

The authors declare no competing interests.

\section{Additional information}

Supplementary information is available for this paper at https://doi.org/10.1038/ s41588-018-0338-y.

Reprints and permissions information is available at www.nature.com/reprints. Correspondence and requests for materials should be addressed to G.C. or E.O. Publisher's note: Springer Nature remains neutral with regard to jurisdictional claims in published maps and institutional affiliations.

(c) The Author(s), under exclusive licence to Springer Nature America, Inc. 2019 


\section{Methods}

Data generation and models. Lymphoma cell lines used in this study (OCILy19, DoHH2, SU-DHL-10, WSU-DLCL2, Toledo, SU-DHL-6, and Karpas422) were authenticated within the past year by short tandem repeat (STR) cell-line authentication analysis (MicroArray, Switzerland) and cultured as described in the Supplementary Note. OCI-Ly19 cells were transduced with lentiviral particles to express EZH2Y646F in tandem with GFP or the empty vector (OCI-Ly19$\left.\mathrm{EZH} 2^{\mathrm{Y} 646 \mathrm{~F}}\right)$. After the initial transduction, 3-5\% of the GFP-positive cells were sorted to obtain a pure population of GFP-positive cells. We confirmed by RNAsequencing (RNA-seq) analysis that only one copy of mutated EZH2 was integrated in the genome.

Hi-C experiments were performed on Karpas422, WSU-DLCL2, OCI-Ly19$\mathrm{EZH} 2^{\mathrm{WT}}$, and OCI-Ly19-EZH2 $2^{\mathrm{Y} 646 \mathrm{~F}}$, treated with $2 \mu \mathrm{M}$ GSK-126 or DMSO for $72 \mathrm{~h}$. Data generation and analysis are detailed in the Supplementary Note. Targeted chromosome conformation capture using unique molecular identifiers (UMI-4C) was performed in Karpas-422 treated with $2 \mu \mathrm{M}$ GSK126 or DMSO for $72 \mathrm{~h}$, as described in ref. ${ }^{53}$. Hi-C and UMI-4C library preparation, contact-map generation, and data normalization were performed as described in the Supplementary Note. A list of the primers used in this study is provided in Supplementary Table 8.

mRNA expression was assessed by means of RNA-seq in OCI-LY19-EZH2 ${ }^{\text {WT }}$, OCI-Ly19-EZH2 ${ }^{\mathrm{Y} 646 \mathrm{~F}}$, and OCI-Ly19-EZH2 ${ }^{\mathrm{Y} 646 \mathrm{~F}}$ after treatment with the EZH2 inhibitor GSK126 for $72 \mathrm{~h}$. ChIP-seq was performed in OCI-Ly19-EZH2 ${ }^{\mathrm{WT}}$ and OCI-Ly19-EZH2 ${ }^{\mathrm{Y} 646 \mathrm{~F}}$, Toledo, and WSU-DLCL2 cells by using a monoclonal antibody to H3K27me3 (Cell Signaling, 9733). H3K27me3 ChIP-seq data for OCI-Ly7, DOHH2, and Karpas422 were obtained from ENCODE, whereas for WSU-DLCL2, data were obtained from the GEO Database (GSE40792). Targeted ChIP validation was performed for FOXO3 and SESN1 on Toledo and WSU-DLCL2 cell lines. All data generation and analyses are detailed in the Supplementary Note.

STORM was used to compare the structure of TAD6.139 between WSUDLCL2 treated with either $2 \mu \mathrm{M}$ GSK126 or DMSO (four independent experiments), and between OCI-Ly19-EZH2 $2^{\mathrm{WT}}$ and OCI-Ly19-EZH2 ${ }^{\mathrm{Y} 646 \mathrm{X}}$ (three independent experiments). The structure of the neutral TAD1.54 was compared between WSU-DLCL2 treated with either $2 \mu \mathrm{M}$ GSK126 or DMSO (two independent experiments). STORM data were generated and analyzed as described in the Supplementary Note.

Mouse model. We isolated E $\mu$-myc transgenic fetal liver cells from embryos at embryonic day 13.5. The HPCs were grown for $4 \mathrm{~d}$ in a specially adapted growth medium (DMEM, IMDM, L-glutamine, pen/strep, FBS, and SCS-stem cell supplement (Whei, IL3, IL6, SCF, and polybrene) and retrovirally transduced with either the vector MLS-GFP containing shRNA for Sesn1 or Foxo3 or the empty vectors. We inoculated genetically modified HPCs into lethally irradiated, syngeneic wild-type recipient animals. Disease onset was monitored by palpation and blood smears. Data were analyzed in Kaplan-Meier format by using the log-rank (Mantel-Cox) test for statistical significance. Transgenic animals were maintained in EPFL SPF animal facility, and all animal studies were approved by Swiss Cantonal authorities (animal license VD2932 and VD2932.1).

Comparison of intrachromosomal Hi-C contact maps. All pairwise comparisons between intrachromosomal contact maps (or matrices) were based on four different metrics: overall matrix correlation by SCC, fraction of corresponding bins assigned to the same compartment (A or B), conservation of TADs, and conservation of significant interactions between bins.

Matrix correlation (stratum-adjusted correlation coefficient). To compare the overall distribution of contacts across intrachromosomal Hi-C maps, we used the SCC, as implemented in the hicrep $\mathrm{R}_{\text {package }}{ }^{24}$ in Bioconductor ( $g e t . s c c$ function, after having trained the smoothing parameter with the htrain function for the range 0:20). After smoothing $\mathrm{Hi}-\mathrm{C}$ matrices with a $2 \mathrm{D}$ mean filter to reduce noise and bias effects, we stratified chromatin contacts according to the genomic distance between interacting loci. The number of strata $K$ is given by the maximal interaction distance divided by the bin size (for example $K=3,840$, for a $192-\mathrm{Mb}$ chromosome binned at $50 \mathrm{~kb}$; here, we chose the chromosome size as the maximal interaction distance). The SCC was then computed as a weighted average of stratum-specific Pearson's correlation coefficients, and it can be interpreted as a classical correlation coefficient with values ranging from -1 , for perfect anticorrelation, to +1 , for perfect correlation.

Fraction of corresponding bins assigned to the same compartment. Compartments were calculated as previously described ${ }^{3}$ : Hi-C contact matrices were binned at $1 \mathrm{Mb}$ by using the pca.hic function from the HiTC R package in Bioconductor, and compartments A and B were defined according to the signs of the values of the first eigenvector retrieved from principal component analysis of the Pearson correlation matrix of the observed over expected Hi-C matrix (when the first eigenvector separated the two chromosome arms, the second eigenvector was used). To compare the compartments between two intrachromosomal matrices, we defined the sign (positive or negative) associated with a specific compartment (A or B) as the one achieving the highest correlation between the eigenvectors of the two datasets. For each pair of 1,000-kb bins corresponding to the same genomic region in the two Hi-C contact matrices, we compared the sign of the corresponding eigenvalue: concordant signs corresponded to bins assigned to the same compartment, opposite signs corresponded to bins in different compartments. A similarity score for each pair of intrachromosomal maps was defined as the fraction of bins that were assigned to the same compartment.

Conservation of TADs. First, TADs were called at $50-\mathrm{kb}$ resolution by using the TopDom $^{26}$ (TopDom function from the TopDom R package with a window size $w=5)$.

Next, we compared the set of TADs identified in two intrachromosomal matrices by computing the concordance of the two corresponding chromosome partitions. For this purpose, we used the measure of concordance (MoC) previously introduced to compare clustering assignment ${ }^{25}$. MoC is defined as follows:

$$
\operatorname{MoC}(\boldsymbol{P}, \boldsymbol{Q})=\left\{\begin{array}{c}
1 \text { if } N_{P}=N_{Q}=1 \\
\frac{1}{\left(\sqrt{N_{P} N_{Q}}-1\right)}\left(\sum_{i=1}^{N_{P}} \sum_{j=1}^{N_{Q}} \frac{\left\|F_{i, j}\right\|^{2}}{\left\|P_{i}\right\|\left\|Q_{j}\right\|}\right) \text { otherwise }
\end{array}\right\}
$$

where $\mathbf{P}$ and $\mathbf{Q}$ are the sets of TADs being compared, including $N_{P}$ and $N_{Q}$ numbers of TADs, respectively. $P_{i}$ and $Q_{j}$ are two individual TADs within $\mathbf{P}$ and $\mathbf{Q}$ having size $\left\|P_{i}\right\|$ and $\left\|Q_{j}\right\|$, respectively, measured in base pairs. Finally, $\left\|F_{i j}\right\|$ corresponds to the size (number of base pairs) of the overlap between the two TADs $P_{i}$ and $Q_{j}$.

Conservation of significant interactions. The statistical significance of chromatin interactions at the bin level was estimated with $\mathrm{HiC}-\mathrm{DC}^{28}$ in 'fixed bin' mode. The bin size was set to $50 \mathrm{~kb}$, with degrees of freedom $(\mathrm{df})=6$ and size $=1.0$. The hg19 mappability file was retrieved by following the link provided in the URLs section. Interactions were called significant if the HiC-DC $q$ value was smaller than 0.05 . Conservation of significant interactions between two given cell lines $\mathrm{cl}$ and $\mathrm{c} 2$ was computed as the overlap between their set of significant interactions as follows:

$$
\text { overlap }=\frac{\# \text { of common s.i. }}{\min \{(\# \text { of s.i. in } c 1),(\# \text { of s.i. in } c 2)\}}
$$

Generation of Hi-C contact maps for replicates and randomized maps for comparison. To build a reference scale of values for the different metrics, we included comparisons between our $\mathrm{Hi}-\mathrm{C}$ maps and randomized $\mathrm{Hi}-\mathrm{C}$ maps, as well as comparisons between independent replicates of the same cell line.

In the comparisons based on the SCC measure and on the fraction of conserved significant interactions, we generated randomized $\mathrm{Hi}-\mathrm{C}$ maps by shuffling count values within each diagonal of the Hi-C matrix, that is by permuting the number of contacts among bins located at the same genomic distance. For the comparison of TAD by MoC, random TAD partitions were generated by shuffling the original set of TADs in a way that preserved the number and the size of the TADs. The resulting random maps were used to estimate the expected similarity score between $\mathrm{Hi}-\mathrm{C}$ experiments performed without any expected structural similarity.

Additionally, for each cell line, we selected and aggregated independent replicates in two groups with similar total number of reads and generated separate contact maps and the list of contacts (interactomes) for each group. The maps and the interactomes were used to estimate the expected similarity score between $\mathrm{Hi}-\mathrm{C}$ experiments performed on the same cell line (that is, expected highest similarity).

Concordance analyses of $\mathrm{H} 3 \mathrm{~K} 27 \mathrm{me} 3$ and transcriptional changes within TADs. A consensus list of TADs conserved among Karpas422, OCI-Ly19-EZH2 ${ }^{\text {WT }}$, OCILy19-EZH2 ${ }^{\mathrm{Y} 646 \mathrm{~F}}$, and WSU-DLCL2 cell lines was determined by using an approach based on weighted interval scheduling (WIS) efficiently solved with a dynamic programming (DP) approach ${ }^{54}$. Briefly, each interval/TAD received a weight according to how conserved it was across cell lines (allowing a tolerance radius of two bins or $100 \mathrm{~kb}$ ), and the goal was to identify a set of nonoverlapping intervals of maximum total weight (details in Supplementary Note). Using this approach, we identified a total of $n=3,773$ consensus TADs.

H3K27me3 comparison among subcompartments. Chromosome subcompartments derived in GM12878 were downloaded from the GEO database (GSE63525). Subcompartment calls were used to partition contact maps generated by our experiments, given the high concordance between $\mathrm{A}$ and $\mathrm{B}$ compartments derived in GM12878 and in our cell lines (Fig. 1c,d). Moreover, subcompartments have been called only in GM12878, given that the procedure requires an extremely high number of reads (documentation in GSE63525). To compare H3K27me3 across subcompartments, we considered the mean $\mathrm{H} 3 \mathrm{~K} 27 \mathrm{me} 3$ levels within each continuous DNA sequence that was assigned to the same subcompartment ( $\mathrm{sc}$ region) and compared the distribution of mean $\mathrm{H} 3 \mathrm{~K} 27 \mathrm{me} 3$ values among $s c$ regions annotated for the same compartment. Unannotated sc regions were excluded from this analysis. H3K27me3 fold changes were computed for each sc 
region through comparing the mean $\mathrm{H} 3 \mathrm{~K} 27$ me3 values in OCI-Ly19-EZH2 ${ }^{\mathrm{Y} 646 \mathrm{~F}}$ versus OCI-Ly19-EZH ${ }^{\text {WT }}$ cells.

Intra-TAD and inter-TAD correlation between H3K27me3 fold changes and levels. Correlations between normalized $\mathrm{H} 3 \mathrm{~K} 27 \mathrm{me} 3$ profiles of OCI-Ly19-EZH2 ${ }^{\mathrm{Y} 646 \mathrm{~F}}$ and OCI-Ly19-EZH2 ${ }^{\text {WT }}$ cells were investigated as previously described ${ }^{23}$. Briefly, each TAD was divided into ten equally spaced bins, as well as two flanking regions of the same size of the TAD. Mean H3K27me3 levels and the fold changes of mean $\mathrm{H} 3 \mathrm{~K} 27 \mathrm{me} 3$ levels in mutated versus wild-type samples within each of these 30 bins were then stored as a row of an $N \times 30$ matrix, where $N$ is the number of TADs. Finally, Spearman correlation coefficients were computed between each pair of columns of this matrix, thus constructing a $30 \times 30$ symmetric pairwise correlation matrix, whose columns 11-20 corresponded to bins within a TAD, whereas columns 1-10 and 21-30 corresponded to bins within upstream and downstream regions, respectively. The central $10 \times 10$ block represented intra-TAD correlations, whereas the $10 \times 10$ blocks above (or below) it and on its right (or left) corresponded to inter-TAD correlations. The correlation of $\mathrm{H} 3 \mathrm{~K} 27 \mathrm{me} 3$ fold changes as a function of distance for intra-TAD and inter-TAD pairs of loci (Fig. 2d) was performed as follows:

1. Each TAD was divided into bins of $25 \mathrm{~kb}$, as well as its two flanking regions (of the same size as the TAD) upstream and downstream; fold changes in each bin were computed as explained above.

2. A distance vector was constructed as a sequence of distances ranging from $25 \mathrm{~kb}$ to $1 \mathrm{Mb}$, each of them separated by $25 \mathrm{~kb}$.

3. For each element $i$ of the distance vector, we extracted all pairs of loci that were separated by a distance equal to $i$; next, we derived an $N \times 2$ matrix storing the $N$ pairs for which both loci were in the same TAD, and we computed the Spearman correlation coefficient between the two columns, which represented the intra-TAD correlation at distance $i$; analogously, we derived an $\mathrm{M} \times 2$ matrix storing the $M$ pairs for which one locus was in the TAD, and the other was in one of the two flanking regions and computed the inter-TAD correlation for distance $i$ in the same way.

4. Finally, we plotted the intra-TAD and inter-TAD correlation versus distance in two different lines.

Concordance between H3K27me3 changes and TADs. H3K27me3 ChIP-seq levels in OCI-Ly19-EZH2 ${ }^{\mathrm{Y} 646 \mathrm{~F}}$ cells and in OCI-Ly19-EZH2 ${ }^{\mathrm{WT}}$ were jointly normalized across the two cell lines, as described in the Supplementary Note. Normalized $\mathrm{H} 3 \mathrm{~K} 27 \mathrm{me} 3$ levels were averaged within each conserved TAD, and the $\log _{2}$ fold change was computed between the two conditions (mutated versus wild type). Density distributions of $\log _{2}$ fold change values were visualized by using the 'density' R function.

Random distributions of $\mathrm{H} 3 \mathrm{~K} 27 \mathrm{me} 3 \mathrm{TAD}$ fold changes were generated by permutation of intervals (bins) of $\mathrm{H} 3 \mathrm{~K} 27 \mathrm{me} 3 \mathrm{read}$ counts. Specifically, for a given size $S$, each chromosome was partitioned into bins of $S$ base pairs, and read counts within each bin were summed. Corresponding bins in $\mathrm{EZH} 2{ }^{\mathrm{Y} 646 \mathrm{~F}}$ and $\mathrm{EZH} 2$ wild-type OCI-Ly19 cells were permuted together to preserve the H3K27me3 ratio between the two conditions for each bin. Bins within each chromosome were permuted separately. TAD fold changes were recalculated as described above. Random distributions were generated with bin sizes ranging from $1 \mathrm{~kb}$ to $2 \mathrm{Mb}$. The number of TADs obtaining a fold change greater than two was compared when using the observed H3K27me3 distribution and distributions generated with bin size ranging from $50 \mathrm{~kb}$ to $2 \mathrm{Mb}$.

Concordance between transcriptional changes and TADs. For each mRNA expression dataset, genes with detectable expression were assigned to the TADs with the highest overlap, and genes nonoverlapping with a TAD were discarded. TADs containing fewer than three genes or more than the 0.99 -quantile of the number of genes by TAD distribution were excluded. With these filters, we retained $n=2,038$ TADs for the comparison between $\mathrm{EZH} 2^{\mathrm{Y} 646 \mathrm{X}}$ and $\mathrm{EZH} 2^{\mathrm{WT}}$ DLBCL primary samples and cell lines, $n=1,923$ TADs for the comparison of follicular lymphoma primary samples, and $n=900$ TADs for the comparison of OCI-Ly19 EZH2 $2^{\mathrm{Y} 646 \mathrm{~F}}$ and OCI-Ly19-EZH2 ${ }^{\mathrm{WT}}$. For the latter comparison, we retained considerably fewer TADs, a result consistent with the RNA-seq dataset including only three replicates for OCI-Ly19-EZH2 ${ }^{\mathrm{Y} 646 \mathrm{~F}}$ and three replicates for OCI-Ly19-EZH2 ${ }^{\text {WT}}$; thus, fewer genes had detectable expression in both conditions than in the other datasets.

To test whether the mRNA expression changes were more concordant within TADs than expected, we first defined TAD mRNA expression fold changes as the mean of the fold changes of each gene within the TAD. Gene fold changes were determined for both microarray and RNA-seq datasets as described above. Next, we defined a measure of concordance for fold changes within a TAD and compared the observed cumulative sum distribution of concordance values obtained by our set of conserved TADs with the expected cumulative sum distribution under random permutation of the gene-toTAD assignments. Briefly, an FCC score was computed for each TAD as:

$$
F C C=\left(2 \frac{\# F C^{-}}{\# F C}-1\right) *\left(2 \frac{\sum\left|F C^{-}\right|}{\sum|F C|}-1\right)
$$

where \#FC is the number of genes within the TAD, $\# \mathrm{FC}^{-}$is the number of genes with a negative fold change within the TAD, $|\mathrm{FC}|$ are absolute fold-change values, and $\mid \mathrm{FC}^{-}$ are absolute values of negative fold changes ( $\log _{2}$ fold changes were used). TADs were ranked from highest to lowest FCC, and the cumulative sum curve was calculated. For each test, we compared the observed cumulative sum curve against curves obtained after random permutation of gene-to-TAD assignments. Intuitively, the more concordant the gene expression changes within TADs, the steeper the increase in the cumulative sum. We considered the observed concordance to be greater than expected when the observed curve was higher than all random curves. To permute gene-toTAD assignments, we divided genes in five classes according to their overall levels of expression and shuffled TAD labels within each class. For each test, we performed 10,000 permutations and reported the range comprising all random curves.

Pearson's correlation of mRNA expression between genes located in the same TAD or different TADs were compared as a function of their genomic distance (maximum $500 \mathrm{~Kb}$ ). Microarray data were normalized by using the median absolute deviation method, whereas RNA-seq data was transformed by using quantile normalization. Curves were fitted by using the $\mathrm{R}$ loess function (from the stats package, with default parameters).

Genes within inactive TADs were analyzed by gene-set enrichment analysis, and their expression was assessed in centrocytes, centroblasts, and memory B-cells, as described in the Supplementary Note.

Differential intra-TAD interactome analysis for TAD6.139. For each pair of cell lines/conditions, significantly different interactions between $20-\mathrm{kb}$ bins within the TAD 6.139 were determined as follows:

1. HiC-DC was run on $\mathrm{Hi}-\mathrm{C}$ read pairs of Chr.6 with a bin size of $20 \mathrm{~kb}$, and the $q$ values obtained for each pair of bins within the entire chromosome were retained;

2. For each pair of bins $i$ and $j$ (with $i_{s>} j_{s}$ ), the interaction difference $d$ between condition $\mathrm{A}$ and condition $\mathrm{B}$ was computed as $d_{i, j}^{A-B}=-\log _{10}\left(q_{i, j}^{A}\right)+\log _{10}\left(q_{i, j}^{B}\right)$;

3. Distributions of $d_{i, j}^{A-B}$ values were determined for pairs of bins at the same genomic distance;

4. For each pair of bins $i_{s}$ and $j_{s}$ in TAD6.139, the significance of the corresponding interaction difference was tested by computing its empirical $P$ value against the distribution of interaction differences computed between bins at the same distance, namely $\left\{d_{i, j}^{A-B}\right\}$

5. Empirical $P$ values obtained for all pairs of bins within TAD 6.139 were corrected for multiple testing by using the Benjamini-Hochberg procedure;

6. Finally, interactions were called significantly stronger in condition A than in condition B if they satisfied both the following conditions:

a. The $q$ value computed by HiC-DC was smaller than 0.1 ,

b. The $q$ value resulting from step (5) was smaller than 0.1 .

Differential-interaction analysis for intra-TAD promoter-proximal regions. For each pair of cell lines/conditions A and B, significantly different interactions between $50-\mathrm{kb}$ bins mapping to gene promoters located in a set of TADs were determined as follows:

1. HiC-DC was run on Hi-C read pairs of all chromosomes with a bin size of $50 \mathrm{~kb}$, and the $q$ values obtained for each pair of bins were retained;

2. Bin-level promoter-promoter interactions (PPIs) between genes $x$ and $y$ were identified as $P P I_{x, y}=-\log _{10}\left(q_{j,}\right)$, where $i$ and $j$ are 50-kb bins overlapping the transcription start sites of genes $x$ and $y$, respectively. Only PPIs between genes in the same TAD and less than $2 \mathrm{Mb}$ apart were retained;

3. Significantly different PPIs between conditions A and B were determined as described in steps 2-6 described in 'Differential intra-TAD interactome analysis', with a cutoff of 0.25 for $q$ values computed in step 5

Random sets of neutral TADs were sampled from our consensus list of 2,038 TADs such that:

1. Only TADs with $\mid \log _{2}(\mathrm{H} 3 \mathrm{~K} 27 \mathrm{me} 3$ fold change $\mathrm{OCI}-L y 19 E Z H 2-Y 646 \mathrm{~F} v \mathrm{EZH} 2-w t) \mid<0.5$ and $\mid \log _{2}(\mathrm{mRNA}$ fold change Cell lines EZH2 $-Y 646 \mathrm{X}$ vs EZH2 -wt $) \mid<0.5$ were sampled;

2. The distributions of the numbers of genes per TAD in the random sets were the same as in the set of 72 inactive TADs

Reporting Summary. Further information on research design is available in the Nature Research Reporting Summary linked to this article.

\section{Data availability}

In this study, we used the following mRNA expression datasets: GSE23501 for wildtype and EZH2-mutated GCB-DLBCL primary human samples, PRJNA278311 (NCBI-BioProject) for wild-type and EZH2-mutated FL primary human samples, GSE40792 for wild-type and EZH2-mutated cell lines before and after treatment with GSK126, GSE49284 for EZH2-mutated cell lines before and after treatment 
with EPZ6438, and GSE12195 for centrocytes, centroblasts, and memory B cells. ChIP-seq data for H3K27me3 in OCI-Ly7, DOHH-2, and Karpas-422 were downloaded from ENCODE; H3K27me3 in WSU-DLCL2 was downloaded from GSE40970; H3K4me3 in OCI-Ly7 and Karpas-422 were downloaded from ENCODE. ChIP-seq data for H3K27me3 and RNA-seq data for OCI-Ly19 and OCI-Ly19-EZH2 ${ }^{\mathrm{Y} 646 \mathrm{~F}}$ were generated as described in the manuscript and have been deposited at GSE114270. HiC matrices and UMI-4C data have been deposited at Zenodo: https://doi.org/10.5281/zenodo.1244182. Custom scripts are available through a public GitHub repository at: https://github.com/CSOgroup/Donaldsonet-al-scripts/.

\section{References}

53. Schwartzman, O. et al. UMI-4C for quantitative and targeted chromosomal contact profiling. Nat. Methods 13, 685-691 (2016).

54. Kleinberg, J. \& Tardos, É. Algorithm Design (Pearson, Boston, 2005). 


\section{natureresearch}

\section{Reporting Summary}

Nature Research wishes to improve the reproducibility of the work that we publish. This form provides structure for consistency and transparency in reporting. For further information on Nature Research policies, see Authors \& Referees and the Editorial Policy Checklist.

\section{Statistical parameters}

When statistical analyses are reported, confirm that the following items are present in the relevant location (e.g. figure legend, table legend, main text, or Methods section).

$\mathrm{n} / \mathrm{a}$ Confirmed

$\bigotimes$ The exact sample size $(n)$ for each experimental group/condition, given as a discrete number and unit of measurement

$\square$ An indication of whether measurements were taken from distinct samples or whether the same sample was measured repeatedly

The statistical test(s) used AND whether they are one- or two-sided

Only common tests should be described solely by name; describe more complex techniques in the Methods section.

\A description of all covariates tested

$\square$ A description of any assumptions or corrections, such as tests of normality and adjustment for multiple comparisons

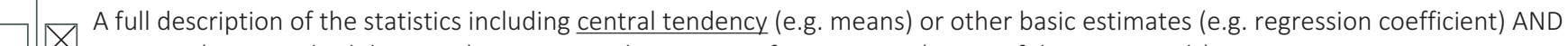

variation (e.g. standard deviation) or associated estimates of uncertainty (e.g. confidence intervals)

$\varnothing$ For null hypothesis testing, the test statistic (e.g. $F, t, r$ ) with confidence intervals, effect sizes, degrees of freedom and $P$ value noted

Give P values as exact values whenever suitable.

Х $\square$ For Bayesian analysis, information on the choice of priors and Markov chain Monte Carlo settings

Х $\square$ For hierarchical and complex designs, identification of the appropriate level for tests and full reporting of outcomes

$\square$ \. Estimates of effect sizes (e.g. Cohen's $d$, Pearson's $r$ ), indicating how they were calculated

$\varnothing$ Clearly defined error bars

State explicitly what error bars represent (e.g. SD, SE, CI)

Our web collection on statistics for biologists may be useful.

\section{Software and code}

Policy information about availability of computer code

Data collection

Data collected from public repository (e.g. ENCODE) was directly retrieved from the associated website without the use of custom software as described in the Methods section.

Flow cytomentry data were acquired using LSR II (BD).

qPCR data were acquired using AB Applied STEPOne PLUS.

sequencing was performed using Illumina $\mathrm{HI}$-seq and NexSeq500.

Histology and immunofluorescence picture were acquired with Leica DM4000 B-LED with Leica application suite v2.0.

Western blot were acquired with LICOR and Image Studio light program v3.1.4

STORM imaging were acquired using a custom build microscope (Douglass et al. Nat.Photonics 2016) but with a photometrics prime sCMOS camera.

Data analysis

Publicly available computational tools to derive TADs, compute the interactome, and process $\mathrm{Hi}-\mathrm{C}$ data matrices were used as described in the Methods section and they include:

Bowtie2-2.2.9

SAMtools (v1.3.1 and v1.6)

Picard tools (v2.5.0)

bwa mem (v0.7.12)

STAR 2.5.3a 
Cufflinks 2.2.1

SRAtools

R package Rsubread 1.28.1

R package limma 3.34.8

SICERpy (wrapper for SICER_V1.1)

IGV genome browser

bedGraphToBigWig v4

R package hicrep $v 1.0 .0$

TopDom v0.0.2

R package HiC-DC v1.0

mSigDB

PRISM 6

FlowJo 10.4.1

Custom scripts have been implemented in bash, R (v3.4.3) and Python (v2.7.5) and are made available through a public GitHub repository at: https://github.com/CSOgroup/Donaldson-et-al-scripts

Finally, standard statistical analyses and related figures were generated using standard R functions as described in the Methods section.

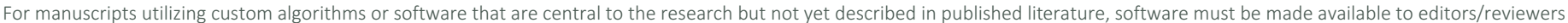

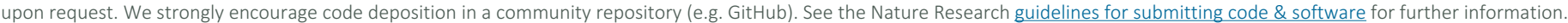

\section{Data}

Policy information about availability of data

All manuscripts must include a data availability statement. This statement should provide the following information, where applicable:

- Accession codes, unique identifiers, or web links for publicly available datasets

- A list of figures that have associated raw data

- A description of any restrictions on data availability

In this study, we used the following mRNA expression datasets: GSE23501 for wild type and EZH2 mutated GC-DLBCL primary human samples and PRJNA278311 for follicular lymphoma primary human samples (Fig. 2), GSE40972 for wild type and EZH2 mutated cell lines before and after treatment with GSK126 (Fig. 2 and Fig. 5), and GSE49284 for EZH2 mutated cell lines before and after treatment with EPZ6438 (Fig. 5). GSE12195 for normal centroblast, centrocytes and memory B-cells mRNA expression (Suppl. Figure 3).

\section{Field-specific reporting}

Please select the best fit for your research. If you are not sure, read the appropriate sections before making your selection.

$\bigotimes$ Life sciences $\quad \square$ Behavioural \& social sciences $\square$ Ecological, evolutionary \& environmental sciences

For a reference copy of the document with all sections, see nature.com/authors/policies/ReportingSummary-flat.pdf

\section{Life sciences study design}

All studies must disclose on these points even when the disclosure is negative.

Sample size Analyses of publicly available datasets used all available samples and multiple independent datasets were used to corroborate the reproducibility of the findings.

The number of animals used for HPC cells experiments $(n>13)$ were in accordance with power analyses done based on previous experiment were $n=10$ was deemed sufficient.

Data exclusions

Differential expression analysis performed only on cell lines from GSE40972 exhibiting hotspot mutations for EZH2 or EZH2 and UTX wild-type. HT cells were excluded as they harbor a mutation in UTX gene. UTX can in part mimic the effect of EZH2 mutations, thus we couldn't include these cells either in the wild-type or in the EZH2 mutant group. Pfeiffer cells were excluded because they harbor a different mutation in EZH2 (A677).Both of these cell lines were per-excluded from the analysis.

In the STORM data analysis, we excluded measurements with extreme numbers of localizations (low or high) which were associated with inflation or deflation of Rg and E values due to technical reasons related to the STORM acquisitions. The detailed procedure is reported in the supplementary note. All measurements are reported in Supplementary Table 7 along with flag indicating whether they were retained or excluded from subsequent analyses.

$\begin{array}{lll}\text { Replication } & \text { Results presented in this study have been confirmed on independent datasets. }\end{array}$

The replication number for each experiment is reported in each figure legend and individual data

Randomization The experimental design did not included a randomization of the samples in different group, however experimental observations have been compared to analytically and empirically derived random models to assess their statistical significance as described in the corresponding method sections.

Blinding

For the animal experiments, we measured survival and cell composition. In the initial experimental design we did not include blinding, as we wanted to match cell composition before and after the injection 


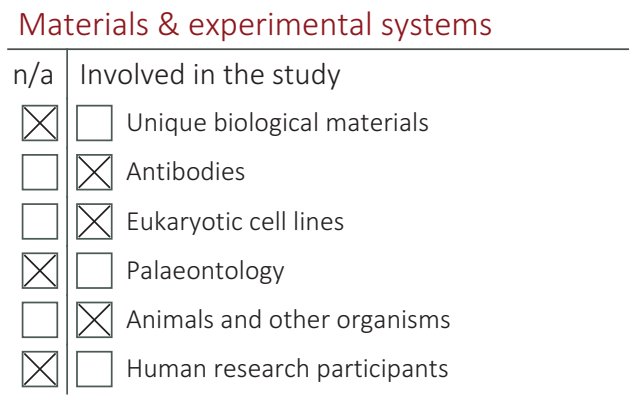

\begin{tabular}{l|l} 
Methods \\
\hline n/a Involved in the study \\
$\square$ & $\bigotimes$ ChIP-seq \\
$\square$ & $\bigotimes$ Flow cytometry \\
$\square$ MRI-based neuroimaging
\end{tabular}

Antibodies

Antibodies used

Primary Antibodies

Tri-methyl histone H3 (Lys 27) (H3K27me3), Rabbit monoclonal antibody (clone C36B11), Cell Signaling 9733; 1:1000 dilution for western blots, 1:1600 dilution for immunofluorescence, and 1:50 for ChIP

Di-methyl histone H3 (Lys 27) (H3K27me2), Rabbit polyclonal antibody Abcam 194690; 1:500 dilution for western blot

Histone H3, XP Rabbit monoclonal antibody (clone D1H2), Cell Signaling 4499; 1:1000 dilution for western blot

Histone H3 (D2B12) XP® Rabbit mAb (ChIP Formulated), Cell Signaling 4620; 1:50 dilution for ChIP

Normal Rabbit IgG, Rabbit polyclonal antibody, Cell Signaling 2729; 1:500 dilution, or 1ug for ChIP

EZH2, Mouse monoclonal antibody (clone AC22), Cell Signaling 3147; 1:1000 dilution for western blots

B-Actin, Mouse monoclonal antibody (clone 8H10D10), Cell Signaling 3700; 1:1000 dilution for western blot

Ki67, Rabbit monoclonal antibody (clone SP6), Abcam Ab16667; 1:200 dilution

CD3 $\alpha$-rat APC (clone 17A2), lot 6224663, BD Farmingen 565643; 1:100 dilution

CD45R/B220 $\alpha$-rat PE-Cy7 (RA3-6B2), lot 6105665, BD Farmingen 552772; 1:100 dilution

Secondary Antibodies

Streptavidin Alexa Fluor-647 conjugate, $\alpha$-biotin, ThermoFischer S21374 (1:500 dilution)

IRDye 800CW Goat anti-Mouse IgG (H + L), 0.1 mg (1:10000 dilution)

IRDye 680RD Goat anti-Mouse IgG (H + L), $0.1 \mathrm{mg}(1: 10000$ dilution)

Goat anti-Rabbit IgG (H+L) Highly Cross-Adsorbed Secondary Antibody, Alexa Fluor 488, Invitrogen (A-11034) (1:500 dilution)

4',6-Diamidino-2-Phenylindole, Dihydrochloride (DAPI) Invitrogen D1306 (1:5000 dilution)

Validation

1. CD45R/B220 $\alpha$-PE-Cy7 and CD3 $\alpha$-APC were used for flow cytometry analyses. Internal negative (unstained) controls were used for all samples.

2. Rabbit $\alpha$-Ki67 (SP6) was used for histological analysis of tumors. Internal negative control (secondary only) stained slides were used for all samples

3. Tri-methyl histone H3 (Lys 27) (H3K27me3), Rabbit IgG (2729) and Histone H3 (4620) were used for ChIP qPCR/ChIP sequencing experiments. These antibodies were used a part of a kit offered by Cell Signaling Technologies (\#9003). The kit contains a positive control Histone H3 Antibody, a negative control Normal Rabbit IgG Antibody and primer sets for PCR detection of the human and mouse ribosomal protein L30 (RPL30) genes. This kit has been extensively used and cited in a number of papers ((1) Orlando, V. (2000) Trends Biochem Sci 25, 99-104., (2) Kuo, M.H. and Allis, C.D. (1999) Methods 19, 42533., (3) Agalioti, T. et al. (2000) Cell 103, 667-78., (4) Soutoglou, E. and Talianidis, I. (2002) Science 295,1901-4., (5) Mikkelsen, T.S. et al. (2007) Nature 448, 553-60., (6) Lee, T.I. et al. (2006) Cell 125, 301-13., (7) Weinmann, A.S. and Farnham, P.J. (2002) Methods 26, 37-47., (8) Wells, J. and Farnham, P.J. (2002) Methods 26, 48-56.).

4. Tri-methyl histone H3 (Lys 27) (H3K27me3) (9733), Di-methyl histone H3 (Lys 27) (H3K27me2) (194690), Histone H3 (4499), EZH2 (3147) and $\beta$-Actin (3700) were used as primary antibodies for western blot analyses. IRDye 800CW Goat anti-Mouse IgG and CD45R/B220 $\alpha$-rat PE-Cy7 were used as secondary antibodies for western blot analyses. 
Histone H3 (4499) has been cited in over 200 peer reviewed journals. Cell Signaling Technology cites no cross reactivity with other histones, and provides a sample western blot for the endogenous detection of Histone $\mathrm{H} 3$ in of four separate cells lines (HeLa, C6, COS, and NIH/3T3).

H3K27me2 (194690) has been cited in 2 peer reviewed journals. Abcam provides a sample western blot for the endogenous detection of H3K27me2 in HeLa cells with no detection observed detection of H3K27me2 in E.coli.

H3K27me3 (9733) has been cited in over 200 peer reviewed journals. Cell Signaling Technology states "Tri-Methyl-Histone H3 (Lys27) (C36B11) Rabbit mAb detects endogenous levels of histone H3 only when tri-methylated on Lys27. The antibody does not cross-react with non-methylated, mono-methylated or di-methylated Lys27. In addition, the antibody does not cross-react with mono-methylated, di-methylated or tri-methylated histone H3 at Lys4, Lys9, Lys36 or Histone H4 at Lys20." Cell signaling provides a sample western blot for the endogenous detection of H3K27me3 in four separate cell lines (HeLa, C6, COS, and $\mathrm{NIH} / 3 \mathrm{~T} 3)$.

EZH2 (3147) has been cited in over 70 peer reviewed journals. Cell signaling provides a sample western blot for the endogenous detection of EZH2 in four separate cell lines (T47D, MCF7, SEM, MDA-MB134).

$\beta$-Actin (3700) has been cited in over 650 peer reviewed journals. Cell signaling provides a sample western blot for the endogenous detection of $\beta$-Actin in five separate cell lines (COS, HeLa, C2C12, C6, CHO).

5. Tri-methyl histone H3 (Lys 27) (H3K27me3) (9733) was used as a primary antibody for immunofluorescence staining (1:1600). Goat anti-Rabbit IgG Alexa Fluor 488 (1:500) (A-11034) and DAPI (D1306) (D1306) (1:5000) were used as secondary antibodies for immunofluorescence stain.

Internal positive and negative (secondary only) control stained slides were used for validation. In addition, Cell Signaling cites over 200 peer reviewed journals where H3K27me3 (9733) has been used, and states that "Tri-Methyl-Histone H3 (Lys27) (C36B11) Rabbit mAb detects endogenous levels of histone $\mathrm{H} 3$ only when tri-methylated on Lys27. The antibody does not crossreact with non-methylated, mono-methylated or di-methylated Lys 27 . In addition, the antibody does not cross-react with monomethylated, di-methylated or tri-methylated histone H3 at Lys4, Lys9, Lys36 or Histone H4 at Lys20." Cell signaling provides an example image of HeLa cells immunolabeled with the (H3K27me3) (9733) antibody. Goat anti-Rabbit IgG Alexa Fluor 488 (A-11034) has been cited in over 500 peer reviewed journals, and uses a process of cross-absorption to remove antibodies that potentially cross react with other species, which increases specificity and reduces background.

6. Streptavidin $\alpha$-biotin Alexa Fluor-647 (1:500) (S21374) and custom designed oligo probes (MYcroarray) were used for DNA FISH and STORM imaging experiments. Internal negative control (secondary only) stained slides were used for validation of the secondary antibody.

\section{Eukaryotic cell lines}

Policy information about cell lines

Cell line source(s)

DoHH2 (ACC-47) DSMZ, WSU-DLCL2 (ACC-575) DSMZ, SU-DHL-10 (ACC-576) DSMZ, OCI-LY19 (ACC-528) DSMZ, Karpas-422 and Toledo and Fl-5-12 cells were obtained from Wendel lab (MSKCC)

Authentication

All human cell lines were authenticated by short tandem repeat (STR) profiling, performed by Microsynth, $\mathrm{CH}$.

Mycoplasma contamination cells obtained for Wendel lab were tested and were negative for mycoplasma contamination, cell bought from DSMZ have not been tested for mycoplasma contamination.

Commonly misidentified lines (See ICLAC register)

No commonly misidentified cell lines were used.

\section{Animals and other organisms}

Policy information about studies involving animals; ARRIVE guidelines recommended for reporting animal research

Laboratory animals

Wild animals

Field-collected samples mus musculus, C57BL6, females, 8-10 weeks old, HPC were isolated from embryo at 13.5-14.5 days.

This study did not involve wild animals

This study did not involve field-collected samples

\section{ChIP-seq}

\section{Data deposition}

Х Confirm that both raw and final processed data have been deposited in a public database such as GEO.

Х Confirm that you have deposited or provided access to graph files (e.g. BED files) for the called peaks. 
Data access links

May remain private before publication.

Files in database submission

Genome browser session

(e.g. $\underline{\text { UCSC) }}$

Methodology

Replicates

Sequencing depth

Antibodies

Peak calling parameters

Data quality

Software
H3K27me3 for WSU-DLCL2 were downloaded from GSE40970

H3K4me3 for OCl-Ly7 and Karpas-422 were downloaded from ENCODE

H3K27me3 and RNAseq for OCI-Ly19-WT and OCI-Ly19-EZH2 Y646F were generated as described in the manuscript and deposited in GSE114270, the token for reviewer access is admhsguudlkldwv

$\mathrm{HiC}$ matrices that can be visualized with Juicebox have been deposited in Zenodo in a private link

https://zenodo.org/record/1244183?

token=eyJhbGciOiJIUzI1NilsImV4cCI6MTUyODU4MTU5OSwiaWFOljoxNTI1OTQ1MjgOfQ.eyJkYXRhljp7InJIY2IkljoxMjQ0MTgzf

SwiaWQiOjUzOSwicm5kljoiYTJhNTIzMjlifQ.3IznpUuQFO-Nkbu895VkhvmUhNieYFFsZOZCqedFuHw

FASTQ and .bam alignment files for H3K27me3 and input (OCI-Ly19-WT and OCI-Ly19-Y646F)

.bed peak lists for H3K27me3 (OCI-Ly19-WT and OCI-Ly19-Y646F)

N/A

OCI-LY19-WT and OCI-LY19-Y646F: 3 replicates per condition

OCI-LY19-WT: 149102952 mapped (MAPQ>0) reads for H3K27me3 ChIP and 48538036 for the input OCI-LY19-Y646F: 122262552 mapped (MAPQ>0) reads for H3K27me3 ChIP and 46698494 for the input

H3K27me3: Cell Signaling 9733

Peaks were called with SICER V1.1, using hg19, fragment size $250 \mathrm{bp}$; effective genome size fraction 0.86; window size 750 bp; gap size 3; redundancy threshold 1; FDR 0.001

- Mapping quality filtering to exclude secondary alignments and reads aligning to multiple locations (MAPQ>0)

- Strict FDR threshold to retain SICER peaks (FDR<0.001)

- Visual inspection of ChIP, input and peak tracks on IGV

- Bowtie2-2.2.9 for the alignment

- Samtools-1.6 for removing PCR duplicates, sorting and filtering

- SICERpy (wrapper for SICER V1.1) for peak calling

- bedGraphToBigWig $\vee 4$ was used to convert bedGraph to bigWig file

- IGV genome browser for visualization

- Custom scripts under https://github.com/CSOgroup/Donaldson-et-al-scripts for downstream analyses

\section{Flow Cytometry}

Plots

Confirm that:

Х The axis labels state the marker and fluorochrome used (e.g. CD4-FITC).

Х The axis scales are clearly visible. Include numbers along axes only for bottom left plot of group (a 'group' is an analysis of identical markers).

Х All plots are contour plots with outliers or pseudocolor plots

Х A numerical value for number of cells or percentage (with statistics) is provided.

Methodology

Sample preparation

Instrument

Software

Cell population abundance

Gating strategy
Cells were retrovirally transduced with either shRNA for Sesn1, Foxo3, or MSCV-IRES-GFP or MSCV-IRES-DsRed vectors. Cells were acquired in the indicated days

The number of fluorescent cells were monitored by FACS (Guava Millipore EasyCyte HT System or LSRII Snoopy , Becton Dickinson).

The data were analyzed using FlowJo.

All cells were considered in the analyses.

In the initial gating $\mathrm{FSC} / \mathrm{SCC}$ we only excluded debris or dead cells that in general accounted for less than $10 \%$ of the population all living cells were considered in the analyses.

Tick this box to confirm that a figure exemplifying the gating strategy is provided in the Supplementary Information. 